JOURNAL OF THE

AMERICAN MATHEMATICAL SOCIETY

Volume 9, Number 2, April 1996

\title{
A BILINEAR ESTIMATE WITH APPLICATIONS TO THE KdV EQUATION
}

\author{
CARLOS E. KENIG, GUSTAVO PONCE, AND LUIS VEGA
}

\section{INTRODUCTION}

In this article we continue our study of the initial value problem (IVP) for the Korteweg-de Vries $(\mathrm{KdV})$ equation with data in the classical Sobolev space $H^{s}(\mathbb{R})$. Thus we consider

$$
\left\{\begin{array}{l}
\partial_{t} u+\partial_{x}^{3} u+\partial_{x}\left(\frac{u^{2}}{2}\right)=0, \quad t, x \in \mathbb{R} \\
u(x, 0)=u_{0}(x)
\end{array}\right.
$$

where $u_{0} \in H^{s}(\mathbb{R})$. Our principal aim here is to lower the best index $s$ for which one has local well posedness in $H^{s}(\mathbb{R})$, i.e. existence, uniqueness, persistence and continuous dependence on the data, for a finite time interval, whose size depends on $\left\|u_{0}\right\|_{H^{s}}$.

Equation in (1.1) was derived by Korteweg and de Vries [21] as a model for long wave propagating in a channel. A large amount of work has been devoted to the existence problem for the IVP (1.1). For instance, (see [9], [10]), the inverse scattering method applies to this problem, and, under appropriate decay assumptions on the data, several existence results have been established, see [5],[6], [14], [28], [33]. Another approach, inherited from hyperbolic problems, relies on the energy estimates, and, in particular shows that (1.1) is locally well posed in $H^{s}(\mathbb{R})$ for $s>3 / 2$, (see [2],[3],[12],[29],[30],[31]). Using these results and conservation laws, global (in time) well posedness in $H^{s}(\mathbb{R}), s \geq 2$ was established, (see [3],[12],[30]). Also, global in time weak solutions in the energy space $H^{1}(\mathbb{R})$ were constructed in [34]. In [13] and [22] a "local smoothing" effect for solutions of (1.1) was discovered. This, combined with the conservation laws, was used in [13] and [22] to construct global in time weak solutions with data in $H^{1}(\mathbb{R})$, and even in $L^{2}(\mathbb{R})$. In [16], we introduced oscillatory integral techniques, to establish local well posedness of (1.1) in $H^{s}(\mathbb{R}), s>3 / 4$, and hence, global (in time) well posedness in $H^{1}(\mathbb{R}), s \geq 1$. (In [16] we showed how to obtain the above mentioned result by Picard iteration in an appropriate function space.) In [4] J. Bourgain introduced new function spaces, adapted to the linear operator $\partial_{t}+\partial_{x}^{3}$, for which there are good "bilinear" estimates for the nonlinear term $\partial_{x}\left(u^{2} / 2\right)$. Using these spaces, Bourgain was able to establish local well posedness of $(1.1)$ in $H^{0}(\mathbb{R})=L^{2}(\mathbb{R})$, and hence, by a conservation

Received by the editors July 13, 1994 and, in revised form, May 11, 1995.

1991 Mathematics Subject Classification. Primary 35Q53; Secondary 35G25, 35D99.

Key words and phrases. Schrödinger equation, initial value problem, well-posedness

C. E. Kenig and G. Ponce were supported by NSF grants. L. Vega was supported by a DGICYT grant. 
law, global well posedness in the same space. The method was first introduced by Bourgain in order to obtain corresponding results in the spatially periodic setting (see (1.2)). These were the first results in the periodic setting, for $s \leq 3 / 2$.

Existence results for weak solutions to (1.1) corresponding to rougher data are also in the literature. Using the inverse scattering method, T. Kappeler [14] showed that if $u_{0}$ is a real measure with appropriate decay at infinity, then (1.1) has a global in time weak solution (see also [5], [6]). Also, Y. Tsutsumi [35] proved the existence of a global, in time, weak solution for $u_{0}$ any positive, finite measure. In [18] we showed that (1.1) is locally well posed in $H^{s}(\mathbb{R})$, for $s>-5 / 8$. Since finite measures are in $H^{s}(\mathbb{R}), s<1 / 2$, this establishes (locally in time) the uniqueness for finite measures. Our method of proof in [18] combined the ideas introduced by Bourgain in [4], with some of the oscillatory integral estimates found in [16] and [17]. This enabled us to extend the bilinear estimate in [18] for $\partial_{x}\left(u^{2} / 2\right)$ to the Bourgain function spaces associated with the index $s$, for $s>-5 / 8$.

In the present paper, we reconsider this bilinear operator in these function spaces, obtaining the best index $s(-3 / 4)$ for which it is bounded. This allows us to obtain the corresponding result for $(1.1)$ in $H^{s}(\mathbb{R})$, for $s>-3 / 4$. Since we also show that our estimates for the bilinear operator are sharp, (except for the limiting case $s=-3 / 4$ which remains open), our result concerning the local well posedness of the IVP (1.1) is the optimal one provided by the method below.

The proof of the estimates for this bilinear operator is based on elementary techniques. In fact, it is similar to those used by C. Fefferman and E. M. Stein [7] to establish the $L^{4 / 3}\left(\mathbb{R}^{2}\right)$ restriction Fourier transform to the sphere and by C. Fefferman [8] to obtain the $L^{4}\left(\mathbb{R}^{2}\right)$ estimate for the Bochner-Riesz operator.

These estimates extend to the periodic case. Thus we can give a simplified proof of J. Bourgain [4] result concerning the local well-posedness of the periodic boundary value problem (PBVP) for the KdV equation

$$
\left\{\begin{array}{l}
\partial_{t} u+\partial_{x}^{3} u+\partial_{x}\left(\frac{u^{2}}{2}\right)=0, \quad t \in \mathbb{R}, x \in \mathbb{T}, \\
u(x, 0)=u_{0}(x)
\end{array}\right.
$$

There is an interesting parallel between the development of the results described above and the recent works of S. Klainerman and M. Machedon, [19] and [20], G. Ponce and T. Sideris [27] and H. Lindblad [23] on non-linear wave equations. In fact, in [19] Klainerman and Machedon consider the IVP

$$
\left\{\begin{array}{l}
\partial_{t}^{2} w^{I}-\Delta w^{I}=N^{I}(w, \nabla w), \quad t \in \mathbb{R}, \quad x \in \mathbb{R}^{3}, \\
w^{I}(x, 0)=f^{I}(x) \in H^{s}\left(\mathbb{R}^{3}\right), \\
\partial_{t} w^{I}(x, 0)=g^{I}(x) \in H^{s-1}\left(\mathbb{R}^{3}\right),
\end{array}\right.
$$

where $w=w^{I}$ is a vector valued function, and the nonlinear terms $N^{I}$ have the form

$$
N^{I}(w, \nabla w)=\sum_{J, K} \Gamma_{J, K}^{I}(w) B_{J, K}^{I}\left(\nabla w^{J}, \nabla w^{K}\right)
$$

where $B_{J, K}^{I}$ is any of the "null forms"

$$
\begin{aligned}
Q_{0}\left(\nabla w^{J}, \nabla w^{K}\right) & =\sum_{i=1}^{3} \partial_{x_{i}} w^{J} \partial_{x_{i}} w^{K}-\partial_{t} w^{J} \partial_{t} w^{K}, \\
Q_{\alpha, \beta}\left(\nabla w^{J}, \nabla w^{K}\right) & =\partial_{x_{\alpha}} w^{J} \partial_{x_{\beta}} w^{K}-\partial_{x_{\beta}} w^{J} \partial_{x_{\alpha}} w^{K},
\end{aligned}
$$


with $0 \leq \alpha \leq \beta \leq 3, \partial_{x_{0}}=\partial_{t}$ and $\Gamma_{J, K}^{I}(w)$ is a polynomial in $w$. Such equations arise in the study of "wave maps" (for $Q_{0}$ ) and Yang-Mills systems in a Coulomb gauge form for the general case.

The energy method establishes the local well posedness of (1.3) if $s>1+3 / 2$. For this result, the special form of the non-linear term is irrelevant. However, Klainerman and Machedon showed in [19], that for the special non-linearities in (1.5), there is a local well posedness for $s \geq 2$. This was done by studying the bilinear operators $Q_{0}(\nabla \phi, \nabla \psi)$ and $Q_{\alpha, \beta}(\nabla \phi, \nabla \psi)$ in (1.5), where $\phi, \psi$ are homogeneous solutions to the linear wave equation, and then extended to the inhomogeneous wave equation via Duhamel's formula. Thus, in this context, the idea of establishing bilinear estimates also appeared, simultaneously to Bourgain's work [4]. Shortly after the work [19], Ponce and Sideris [27] were able to show, using oscillatory integral estimates (the so called "Strichartz estimates" [32], see also [26]), that for "any" quadratic non-linearity $N^{I}$, one has local well posedness in (1.3), for $s>2$. Moreover, Lindblad [23] has shown that for general quadratic non-linearity $N^{I}$, this is sharp. Thus, to achieve $s=2$, the null forms (1.5) are needed. Very recently, Klainerman and Machedon [20], motivated by the results in [4], [18] and those in the present paper, have established better estimates for $Q_{0}(\nabla \phi, \nabla \psi)$, in function spaces somewhat similar to those studied here, but adapted to the wave operator, and have used this to show local well posedness for (1.3) for $s>3 / 2$, when $N^{I}$ involves only $Q_{0}$. The exponent $s=3 / 2$ is sharp.

To state our main results we recall the following definitions.

Definition $1.1([4])$. For $s, b \in \mathbb{R}, X_{s, b}$ denotes the completion of the Schwartz space $S\left(\mathbb{R}^{2}\right)$ with respect to the norm

$$
\|F\|_{X_{s, b}}=\left(\int_{-\infty}^{\infty} \int_{-\infty}^{\infty}\left(1+\left|\tau-\xi^{3}\right|\right)^{2 b}(1+|\xi|)^{2 s}|\widehat{F}(\xi, \tau)|^{2} d \xi d \tau\right)^{1 / 2}
$$

Let $\mathcal{Y}$ be the space of all $F$ such that

i) $F: \mathbb{T} \times \mathbb{R} \rightarrow \mathbb{C}$.

ii) $F(x, \cdot) \in S(\mathbb{R})$ for each $x \in \mathbb{T}$.

iii) $x \rightarrow F(x, \cdot)$ is $C^{\infty}$.

iv) $\widehat{F}(0, \tau)=0$ for all $\tau \in \mathbb{R}$.

For $s, b \in \mathbb{R}, Y_{s, b}$ denotes the completion of $\mathcal{Y}$ with respect to the norm

$$
\|F\|_{Y_{s, b}}=\left(\sum_{n \neq 0} \int_{-\infty}^{\infty}\left(1+\left|\tau-n^{3}\right|\right)^{2 b}|n|^{2 s}|\widehat{F}(n, \tau)|^{2} d \tau\right)^{1 / 2}
$$

For $F \in X_{s, b}$ (or $F \in Y_{s, b}$ ) we define the bilinear operator

$$
B(F, F)(x, t)=\frac{1}{2} \partial_{x}\left(F^{2}(x, t)\right)
$$

Our main results in this work are gathered in the following theorems. 
Theorem 1.1. Given $s \in(-3 / 4,0]$ there exists $b \in(1 / 2,1)$ such that

$$
\|B(F, F)\|_{X_{s, b-1}} \leq c\|F\|_{X_{s, b}}^{2}
$$

Theorem 1.2. For $s \in(-1 / 2,0]$ and $b=1 / 2$ it follows that

$$
\|B(F, F)\|_{Y_{s, b-1}} \leq c\|F\|_{Y_{s, b}}^{2} .
$$

Theorem 1.3. For any $s<-3 / 4$ and any $b \in \mathbb{R}$ the estimate (1.9) fails.

Theorem 1.4. For any $s<-1 / 2$ and any $b \in \mathbb{R}$ the estimate (1.10) fails.

As a consequence of the estimates (1.9)-(1.10) we obtain the following results concerning the IVP (1.1) and the PBVP (1.2).

Theorem 1.5. Let $s \in(-3 / 4,0]$. Then there exists $b \in(1 / 2,1)$ such that for any $u_{0} \in H^{s}(\mathbb{R})$ there exist $T=T\left(\left\|u_{0}\right\|_{H^{s}}\right)>0$ (with $T(\rho) \rightarrow \infty$ as $\rho \rightarrow 0$ ) and a unique solution $u(t)$ of the IVP (1.1) in the time interval $[-T, T]$ satisfying

$$
\begin{gathered}
u \in C\left([-T, T]: H^{s}(\mathbb{R})\right), \\
u \in X_{s, b} \subseteq L_{x, \ell \text { oc }}^{p}\left(\mathbb{R}: L_{t}^{2}(\mathbb{R})\right), \quad \text { for } \quad 1 \leq p \leq \infty, \\
\partial_{x}\left(u^{2}\right) \in X_{s, b-1}
\end{gathered}
$$

and

$$
\partial_{t} u \in X_{s-3, b-1}
$$

Moreover, for any $T^{\prime} \in(0, T)$ there exists $R=R\left(T^{\prime}\right)>0$ such that the map $\widetilde{u}_{0} \mapsto \widetilde{u}(t)$ from $\left\{\widetilde{u}_{0} \in H^{s}(\mathbb{R}):\left\|u_{0}-\widetilde{u}_{0}\right\|_{H^{-s}}<R\right\}$ into the class defined by (1.8)-(1.9) with $T^{\prime}$ instead of $T$ is Lipschitz.

In addition, if $u_{0} \in H^{s^{\prime}}(\mathbb{R})$ with $s^{\prime}>s$, then the above results hold with $s^{\prime}$ instead of $s$ in the same time interval $[-T, T]$.

Theorem 1.6. Let $s \in(-1 / 2,0]$. Then for any $u_{0} \in H^{s}(\mathbb{T})$ with $\hat{u}_{0}(0)=0$ there exist $T=T\left(\left\|u_{0}\right\|_{H^{s}}\right)>0$ and a unique solution $u(t)$ of the PBVP (1.2) in the time interval $[-T, T]$ satisfying

$$
u \in C\left([-T, T]: H^{s}(\mathbb{T})\right) \cap Y_{s, b},
$$

and

$$
\partial_{x}\left(u^{2}\right) \in Y_{s, b-1}
$$

Moreover, for any $T^{\prime} \in(0, T)$ there exists $r=r\left(T^{\prime}\right)>0$ such that the map $\widetilde{u}_{0} \mapsto \widetilde{u}(t)$ from $\left\{\widetilde{u}_{0} \in H^{s}(\mathbb{T}):\left\|u_{0}-\widetilde{u}_{0}\right\|_{H^{-s}}<R\right\}$ into the class defined by (1.12) with $T^{\prime}$ instead of $T$ is Lipschitz.

Once the key estimate (1.10) has been established, the proof of Theorem 1.6, as well as its general version, i.e. for data $u_{0}$ with arbitrary mean value, follows the arguments in [4], therefore it will be omitted. 
Theorem 1.5 improves the results in [4], $s \geq 0$, and that in [18], $s>-5 / 8$. In these works the bounds of the bilinear operator (1.8) in the spaces $X_{s, b}$, were based on several $L^{p} L^{q}$-estimates, i.e. Strichartz type [32], a sharp version of Kato smoothing effect [13] found in [15], maximal functions and those obtained by interpolating these. As it was remarked above our proof is elementary. Indeed, it only uses simple calculus arguments.

Theorem 1.6 was essentially proven in [4]. There the bound for the bilinear operator (1.8) was based on the Zygmund result [36] concerning the restriction of Fourier series in $L^{4}\left(\mathbb{T}^{2}\right)$. As in the continuous case our proof of this key estimate is based on simple calculus inequalities.

It is interesting to compare the above result for the $\mathrm{KdV}$ equation with those obtained, by different arguments, for the modified $\mathrm{KdV}(\mathrm{mKdV})$ equation

$$
\partial_{t} v+\partial_{x}^{3} v+\frac{1}{3} \partial_{x}\left(v^{3}\right)=0
$$

It was proven in [17] that $s \geq 1 / 4$ suffices for the local well posedness of the corresponding IVP of the $\mathrm{mKdV}$ in $H^{s}(\mathbb{R})$. Similar result, with $s>1 / 2$, for the PBVP associated to (1.14) can be found in [4].

We will show that the method used here does not improve these results. More precisely, let

$$
\widetilde{B}(F, F, F)(x, t)=\frac{1}{3} \partial_{x}\left(F^{3}(x, t)\right)
$$

denote the tri-linear operator associated to the $\mathrm{mKdV}$.

Theorem 1.7. For any $s<1 / 4$ and any $b \in \mathbb{R}$ the estimate

$$
\|\widetilde{B}(F, F, F)\|_{X_{s, b-1}} \leq c\|F\|_{X_{s, b}}^{3}
$$

fails.

Theorem 1.8. For any $s<1 / 2$ and any $b \in \mathbb{R}$ the estimate

$$
\|\widetilde{B}(F, F, F)\|_{Y_{s, b-1}} \leq c\|F\|_{Y_{s, b}}^{3}
$$

fails.

We observe that in both cases, the IVP and the PBVP, the difference between the best Sobolev exponents known for the local well posedness of the KdV and the $\mathrm{mKdV}$ is one. Heuristically, this is consistent with the Miura transformation [25] which affirms that if $v(\cdot)$ solves the $\mathrm{mKdV}(1.14)$, then

$$
u=c i \partial_{x} v+v^{2}
$$

solves the $\mathrm{KdV}$ equation.

For the generalized KdV equation

$$
\partial_{t} u+\partial_{x}^{3} u+\frac{1}{k+1} \partial_{x}\left(u^{k+1}\right)=0, \quad x, t \in \mathbb{R}, k \in \mathbb{Z}^{+},
$$


one has that if $u(x, t)$ is a solution, then for any $\lambda>0$

$$
u_{\lambda}(x, t)=\lambda^{2 / k} u\left(\lambda x, \lambda^{3} t\right)
$$

also solves (1.19). This scaling argument suggests the value $s_{k}=(k-4) / 2 k$ as a lower bound for the Sobolev exponent which guarantees the local well posedness of the corresponding IVP. For higher nonlinearity, $k \geq 4$, this lower bound was achieved in [17]. Moreover, it was shown in [1] that this is the best possible result. However, this is not always the case; the following example shows that the values of the Sobolev exponent given by the scaling argument and the optimal one may be different even for positive values. As before, consider the IVP for a type of nonlinear wave equations

$$
\left\{\begin{array}{l}
\partial_{t}^{2} \omega-\Delta \omega=\left(\partial_{t} \omega\right)^{j}, \quad t \in \mathbb{R}, x \in \mathbb{R}^{3}, j=1,2,3, \ldots \\
\omega(x, 0)=f(x) \in H^{s}\left(\mathbb{R}^{3}\right) \\
\partial_{t} \omega(x, 0)=g(x) \in H^{s-1}\left(\mathbb{R}^{3}\right)
\end{array}\right.
$$

The scaling argument suggests that (1.21) is locally well posed for $s>s(j)=$ $(5 j-7) /(2 j-2)$. When $j \geq 3$ this result, as well as its sharpness, was established in [27]. For $j=2$ it was proven in [27] that $s>2$ is sufficient for the local existence and in [23] that $s \geq 2$ is necessary (see also [24]). Observe that in this case, $j=2$, $s(2)=3 / 2$. As was noted before, in [20] it was shown that $s>s(2)=3 / 2$ is actually attained for the null form $Q_{0}$ in (1.5).

This paper is organized as follows. Sections 2-4 are concerned with the nonperiodic case. Theorems 1.1 and 1.5 will be proven in Sections 2 and 3 respectively. Section 4 contains the proof of Theorems 1.3, 1.7. The periodic case is considered in Sections 5-6. Theorem 1.2 will be proven in Section 5, and Theorems 1.4, 1.8 in Section 6 .

\section{Proof of Theorem 1.1}

First we will rewrite the estimate (1.9) in an equivalent form. Defining

$$
\rho=-s \in[0,3 / 4)
$$

it follows from (1.6) that if $F \in X_{s, b}=X_{-\rho, b}$, then

$$
f(\xi, \tau)=\left(1+\left|\tau-\xi^{3}\right|\right)^{b}(1+|\xi|)^{-\rho} \widehat{F}(\xi, \tau) \in L^{2}\left(\mathbb{R}^{2}\right)
$$

and

$$
\|f\|_{L_{\xi}^{2} L_{\tau}^{2}}=\|F\|_{X_{s, b}}=\|F\|_{X_{-\rho, b}}
$$

Using that

$$
\left.\widehat{\partial_{x}\left(F^{2}\right.}\right)(\xi, \tau)=c \xi(\widehat{F} * \widehat{F})(\xi, \tau)
$$


we can rewrite (1.9) in terms of $f$ as

$$
\begin{aligned}
& \left.\|B(F, F)\|_{X_{s, b-1}}=\|\left(1+\left|\tau-\xi^{3}\right|\right)^{b-1}(1+|\xi|)^{-\rho} \widehat{\partial_{x}\left(F^{2}\right.}\right) \|_{L_{\xi}^{2} L_{\tau}^{2}} \\
& =c\left\|\left(1+\left|\rho-\xi^{3}\right|\right)^{b-1}(1+|\xi|)^{-\rho} \xi(\widehat{F} * \widehat{F})\right\|_{L_{\xi}^{2} L_{\tau}^{2}} \\
& =c \| \frac{\xi}{\left(1+\left|\tau-\xi^{3}\right|\right)^{1-b}(1+|\xi|)^{\rho}} \times \\
& \iint \frac{f\left(\xi_{1}, \tau_{1}\right)\left(1+\left|\xi_{1}\right|\right)^{\rho}}{\left(1+\left|\tau_{1}-\xi_{1}^{3}\right|\right)^{b}} \frac{f\left(\xi-\xi_{1}, \tau-\tau_{1}\right)\left(1+\left|\xi-\xi_{1}\right|\right)^{\rho} d \xi_{1} d \tau_{1}}{\left(1+\left|\tau-\tau_{1}-\left(\xi-\xi_{1}\right)^{3}\right|\right)^{b}} \|_{L_{\xi}^{2} L_{\tau}^{2}} \\
& \leq c\|F\|_{X_{s, b}}^{2}=c\|f\|_{L_{\xi}^{2} L_{\tau}^{2}}^{2} .
\end{aligned}
$$

Thus, Theorem 1.1 can be restated as follows.

Theorem 2.1. Given $\rho=-s \in[0,3 / 4)$ there exists $b \in(1 / 2,1)$ such that

$$
\begin{aligned}
& \| \frac{\xi}{\left(1+\left|\tau-\xi^{3}\right|\right)^{1-b}(1+|\xi|)^{\rho}} \times \\
& \iint \frac{f\left(\xi_{1}, \tau_{1}\right)\left(1+\left|\xi_{1}\right|\right)^{\rho}}{\left(1+\left|\tau_{1}-\xi_{1}^{3}\right|\right)^{b}} \frac{f\left(\xi-\xi_{1}, \tau-\tau_{1}\right)\left(1+\left|\xi-\xi_{1}\right|\right)^{\rho}}{\left(1+\left|\tau-\tau_{1}-\left(\xi-\xi_{1}\right)^{3}\right|\right)^{b}} d \xi_{1} d \tau_{1} \|_{L_{\xi}^{2} L_{\tau}^{2}} \\
& \leq c\|f\|_{L_{\xi}^{2} L_{\tau}^{2}}^{2} .
\end{aligned}
$$

We will restrict ourselves to the most interesting cases of $(2.6), \rho=0$, which provides a simplified proof of the $L^{2}$-result in [4], and $\rho \in(1 / 2,3 / 4)$. In fact we will prove the following slightly stronger version of Theorem 2.1.

Theorem 2.2. Given $\rho=-s \in(1 / 2,3 / 4)$ there exists $b \in(1 / 2,1)$ such that for any $b^{\prime} \in(1 / 2, b]$ with $b-b^{\prime} \leq \min \{\rho-1 / 2 ; 1 / 4-\rho / 3\}$ it follows that

$$
\begin{aligned}
& \| \frac{\xi}{\left(1+\left|\tau-\xi^{3}\right|\right)^{1-b}(1+|\xi|)^{\rho}} \times \\
& \iint \frac{f\left(\xi_{1}, \tau_{1}\right)\left(1+\left|\xi_{1}\right|\right)^{\rho}}{\left(1+\left|\tau_{1}-\xi_{1}^{3}\right|\right)^{b^{\prime}}} \frac{f\left(\xi-\xi_{1}, \tau-\tau_{1}\right)\left(1+\left|\xi-\xi_{1}\right|\right)^{\rho}}{\left(1+\left|\tau-\tau_{1}-\left(\xi-\xi_{1}\right)^{3}\right|\right)^{b^{\prime}}} d \xi_{1} d \tau_{1} \|_{L_{\xi}^{2} L_{\tau}^{2}} \\
& \quad \leq c\|f\|_{L_{\xi}^{2} L_{\tau}^{2}}^{2},
\end{aligned}
$$

where the constant $c$ depends on $\rho, b$ and $b-b^{\prime}$.

Moreover (2.7) still holds for $\rho=0, b \in(1 / 2,3 / 4]$ and $b^{\prime} \in(1 / 2, b]$.

We observe that (2.6) follows from (2.7) by taking $b^{\prime}=b$.

The following elementary calculus inequalities will provide the main tool in the proof of Theorem 2.2. 
Lemma 2.3. If $\ell>1 / 2$, then there exists $c>0$ such that

$$
\int_{-\infty}^{\infty} \frac{d x}{(1+|x-\alpha|)^{2 \ell}(1+|x-\beta|)^{2 \ell}} \leq \frac{c}{(1+|\alpha-\beta|)^{2 \ell}}
$$

$$
\int_{-\infty}^{\infty} \frac{d x}{(1+|x|)^{2 \ell}|\sqrt{\alpha-x}|} \leq \frac{c}{(1+|\alpha|)^{1 / 2}}
$$

$$
\int_{-\infty}^{\infty} \frac{d x}{(1+|x-\alpha|)^{2(1-\ell)}(1+|x-\beta|)^{2 \ell}} \leq \frac{c}{(1+|\alpha-\beta|)^{2(1-\ell)}}
$$

and

$$
\int_{|x| \leq \beta} \frac{d x}{(1+|x|)^{2(1-\ell)}|\sqrt{\alpha-x}|} \leq c \frac{(1+\beta)^{2(\ell-1 / 2)}}{(1+|\alpha|)^{1 / 2}} .
$$

Next, using these calculus inequalities, we deduce three lemmas from which the proof of Theorem 2.2 will follow directly.

Lemma 2.4. If $b \in(1 / 2,3 / 4]$ and $b^{\prime} \in(1 / 2, b]$, then there exists $c>0$ such that

$$
\frac{|\xi|}{\left(1+\left|\tau-\xi^{3}\right|\right)^{1-b}} \times
$$

$$
\left(\int_{-\infty}^{\infty} \int_{-\infty}^{\infty} \frac{d \tau_{1} d \xi_{1}}{\left(1+\left|\tau_{1}-\xi_{1}^{3}\right|\right)^{2 b^{\prime}}\left(1+\left|\tau-\tau_{1}-\left(\xi-\xi_{1}\right)^{3}\right|\right)^{2 b^{\prime}}}\right)^{1 / 2} \leq c .
$$

Proof. Since $b^{\prime}>1 / 2$, from (2.8) it follows that

$$
\begin{aligned}
& \int_{-\infty}^{\infty} \frac{d \tau_{1}}{\left(1+\left|\tau_{1}-\xi_{1}^{3}\right|\right)^{2 b^{\prime}}\left(1+\left|\tau-\tau_{1}-\left(\xi-\xi_{1}\right)^{3}\right|\right)^{2 b^{\prime}}} \\
& \leq \frac{c}{\left(1+\left|\tau-\xi^{3}+3 \xi \xi_{1}\left(\xi-\xi_{1}\right)\right|^{2 b^{\prime}}\right)} .
\end{aligned}
$$

To integrate with respect to $\xi_{1}$ we change variables

$$
\mu=\lambda-\xi^{3}+3 \xi \xi_{1}\left(\xi-\xi_{1}\right), \quad \text { then } \quad d \mu=3 \xi\left(\xi-2 \xi_{1}\right) d \xi_{1},
$$

and

$$
\xi_{1}=\frac{1}{2}\left\{\xi \pm \sqrt{\frac{4 \tau-\xi^{3}-4 \mu}{3 \xi}}\right\}
$$


Therefore

$$
\left|\xi\left(\xi-2 \xi_{1}\right)\right|=c \sqrt{|\xi|}\left|\sqrt{4 \tau-\xi^{3}-4 \mu}\right|
$$

and

$$
d \xi_{1}=c \frac{d \mu}{\sqrt{|\xi|} \mid \sqrt{4 \tau-\xi^{3}-4 \mu \mid}} .
$$

Now combining these identities with $(2.9)$ we find

$$
\begin{gathered}
\int_{-\infty}^{\infty} \frac{d \xi_{1}}{\left(1+\left|\tau-\xi^{3}+3 \xi \xi_{1}\left(\xi-\xi_{1}\right)\right|\right)^{2 b^{\prime}}} \\
\leq \frac{c}{\sqrt{|\xi|}} \int_{-\infty}^{\infty} \frac{d \mu}{(1+|\mu|)^{2 b^{\prime}} \mid \sqrt{4 \tau-\xi^{3}-4 \mu \mid}} \leq \frac{c}{|\xi|^{1 / 2}\left(1+\left|4 \tau-\xi^{3}\right|\right)^{1 / 2}} .
\end{gathered}
$$

Thus the term in (2.10) is bounded by

$$
\frac{c|\xi|^{3 / 4}}{\left(1+\left|\tau-\xi^{3}\right|\right)^{1-b}} \frac{1}{\left(1+\left|4 \tau-\xi^{3}\right|\right)^{1 / 4}} .
$$

It is easy to check that the expression in (2.17) is bounded whenever $b \leq 3 / 4$.

Lemma 2.5. If $\rho=-s \in(1 / 2,3 / 4), b \in(1 / 2,3 / 4+s / 3]$ and $b^{\prime} \in\left(\frac{1}{2}, b\right]$, then there exists $c>0$ such that

$$
\frac{|\xi|}{\left(1+\left|\tau-\xi^{3}\right|\right)^{1-b}(1+|\xi|)^{\rho}} \times
$$

$$
\left(\iint_{A} \frac{\left|\xi_{1}\left(\xi-\xi_{1}\right)\right|^{2 \rho}}{\left(1+\left|\tau_{1}-\xi_{1}^{3}\right|\right)^{2 b^{\prime}}\left(1+\left|\tau-\tau_{1}-\left(\xi-\xi_{1}\right)^{3}\right|\right)^{2 b^{\prime}}} d \tau_{1} d \xi_{1}\right)^{1 / 2}<c
$$

where $A=A(\xi, \tau)$ is defined as

$$
\begin{aligned}
A= & \left\{\left(\xi_{1}, \tau_{1}\right) \in \mathbb{R}^{2}|| \xi_{1}|\geq 1,| \xi-\xi_{1} \mid \geq 1,\right. \\
& \left.\left|\tau-\tau_{1}-\left(\xi-\xi_{1}\right)^{3}\right| \leq\left|\tau_{1}-\xi_{1}^{3}\right| \leq\left|\tau-\xi^{3}\right|\right\} .
\end{aligned}
$$

Proof. First we observe that in $A$

$$
\left|\tau-\xi^{3}+3 \xi \xi_{1}\left(\xi-\xi_{1}\right)\right|=\left|\tau_{1}-\xi_{1}+\tau-\tau_{1}-\left(\xi-\xi_{1}\right)^{3}\right| \leq 2|\tau-\xi|^{3} .
$$

Thus, by (2.8)

$$
\begin{aligned}
& \int \frac{d \tau_{1}}{\left(1+\left|\tau_{1}-\xi_{1}^{3}\right|\right)^{2 b^{\prime}}\left(1+\left|\tau-\tau_{1}-\left(\xi-\xi_{1}\right)^{3}\right|\right)^{2 b^{\prime}}} \\
& \leq \frac{\chi\left(\left(\tau-\xi^{3}+3 \xi \xi_{1}\left(\xi-\xi_{1}\right)\right) / 2\left(\tau-\xi^{3}\right)\right)}{\left(1+\left|\tau-\xi^{3}+3 \xi \xi_{1}\left(\xi-\xi_{1}\right)\right|\right)^{2 b^{\prime}}},
\end{aligned}
$$


where $\chi(\cdot)$ denotes the characteristic function of the interval $[-1,1]$.

As in the previous Lemma we use the change of variable

$$
\mu=\tau-\xi^{3}+3 \xi \xi_{1}\left(\xi-\xi_{1}\right)
$$

Thus (2.14)-(2.17), (2.23) and (2.29) lead to the inequalities

$$
\begin{aligned}
& \iint_{A} \frac{\left|\xi_{1}\left(\xi-\xi_{1}\right)\right|^{2 \rho}}{\left(1+\left|\tau_{1}-\xi_{1}^{3}\right|\right)^{2 b^{\prime}}\left(1+\left|\tau-\tau_{1}-\left(\xi-\xi_{1}\right)^{3}\right|\right)^{2 b^{\prime}}} d \xi_{1} d \tau_{1} \\
& \leq c \int \frac{\left|\xi_{1}\left(\xi-\xi_{1}\right)\right|^{2 \rho} \chi\left(\left(\tau-\xi^{3}+3 \xi \xi_{1}\left(\xi-\xi_{1}\right)\right) / 2\left(\tau-\xi^{3}\right)\right)}{\left(1+\left|\tau-\xi^{3}+3 \xi \xi_{1}\left(\xi-\xi_{1}\right)\right|\right)^{2 b^{\prime}}} d \xi_{1} \\
& \leq c \int_{|\mu| \leq 2\left|\tau-\xi^{3}\right|} \frac{\left|\tau-\xi^{3}-\mu\right|^{2 \rho}}{|\xi|^{2 \rho+1 / 2}(1+|\mu|)^{2 b^{\prime}} \mid \sqrt{4 \tau-\xi^{3}-4 \mu \mid}} d \mu \\
& \leq \frac{c}{|\xi|^{2 \rho+1 / 2}} \frac{\left|\tau-\xi^{3}\right|^{2 \rho}}{\left(1+\left|4 \tau-\xi^{3}\right|\right)^{1 / 2}} .
\end{aligned}
$$

Hence the expression

$$
\phi(\xi, \tau)=\frac{c|\xi|^{3 / 4-\rho}}{(1+|\xi|)^{\rho}} \frac{\left(1+\left|\tau-\xi^{3}\right|\right)^{\rho+b-1}}{\left(1+\left|4 \tau-\xi^{3}\right|\right)^{1 / 4}}
$$

bounds the term in (2.20). From the assumptions on $\rho=-s$ and $b$ one has that

$$
\phi \in L^{\infty}\left(\mathbb{R}^{2}\right)
$$

which completes the proof.

Lemma 2.6. If $\rho=-s \in(1 / 2,3 / 4), b \in(1 / 2,1)$ and $b^{\prime} \in(1 / 2, b]$ with $b-b^{\prime} \leq$ $\min \{\rho-1 / 2 ; 1 / 4-\rho / 3\}$, then there exists $c>0$ such that

$$
\frac{1}{\left(1+\left|\tau_{1}-\xi_{1}^{3}\right|\right)^{b^{\prime}}} \times
$$

$$
\left(\iint_{B} \frac{|\xi|^{2(1-\rho)}\left|\xi \xi_{1}\left(\xi-\xi_{1}\right)\right|^{2 \rho} d \tau d \xi}{(1+|\xi|)^{2 \rho}\left(1+\left|\tau-\xi^{3}\right|\right)^{2(1-b)}\left(1+\left|\tau-\tau_{1}-\left(\xi-\xi_{1}\right)^{3}\right|\right)^{2 b^{\prime}}}\right)^{1 / 2} \leq c
$$

where $B=B\left(\xi_{1}, \tau_{1}\right)$ is defined as

$$
\begin{aligned}
B= & \left\{(\xi, \tau) \in \mathbb{R}^{2}|| \xi-\xi_{1}|\geq 1,| \xi_{1} \mid \geq 1,\right. \\
& \left.\left|\tau-\tau_{1}-\left(\xi-\xi_{1}\right)^{3}\right| \leq\left|\tau_{1}-\xi_{1}^{3}\right|,\left|\tau-\xi^{3}\right| \leq\left|\tau_{1}-\xi_{1}^{3}\right|\right\} .
\end{aligned}
$$


Proof. First we observe that in $B$

(2.30) $\quad\left|\tau_{1}-\xi_{1}^{3}+3 \xi \xi_{1}\left(\xi-\xi_{1}\right)\right|=\left|\tau-\xi^{3}-\left(\tau-\tau_{1}-\left(\xi-\xi_{1}\right)^{3}\right)\right| \leq 2\left|\tau_{1}-\xi_{1}^{3}\right|$.

By (2.10) it follows that

$$
\begin{aligned}
& \int \frac{d \tau}{\left(1+\left|\tau-\xi^{3}\right|\right)^{2(1-b)}\left(1+\left|\tau-\tau_{1}-\left(\xi-\xi_{1}\right)^{3}\right|\right)^{2 b^{\prime}}} \\
& \leq \frac{c}{\left(1+\left|\tau_{1}-\xi_{1}^{3}+3 \xi \xi_{1}\left(\xi-\xi_{1}\right)\right|\right)^{2(1-b)}} .
\end{aligned}
$$

Thus to obtain (2.28) it suffices to bound

$$
\begin{aligned}
I(D) & =\frac{1}{\left(1+\left|\tau_{1}-\xi_{1}^{3}\right|\right)^{b^{\prime}}} \times \\
& \left(\int_{D} \frac{|\xi|^{2(1-\rho)}\left|\xi \xi_{1}\left(\xi-\xi_{1}\right)\right|^{2 \rho}}{(1+|\xi|)^{2 \rho}\left(1+\left|\tau_{1}-\xi_{1}^{3}+3 \xi \xi_{1}\left(\xi-\xi_{1}\right)\right|\right)^{2(1-b)}} d \xi\right)^{1 / 2}
\end{aligned}
$$

with $D=B^{\prime}=B^{\prime}\left(\xi_{1}, \tau_{1}\right)$, where

$$
\begin{aligned}
B^{\prime}= & \left\{\xi \in \mathbb{R}|1 \leq| \xi-\xi_{1}|, 1 \leq| \xi_{1}|,| \tau-\xi^{3}|\leq| \tau_{1}-\xi_{1}^{3} \mid\right. \\
& \left.\left|\tau_{1}-\xi_{1}^{3}+3 \xi \xi_{1}\left(\xi-\xi_{1}\right)\right| \leq 2\left|\tau_{1}-\xi_{1}^{3}\right|\right\} .
\end{aligned}
$$

We split $B^{\prime}$ into two subdomains $B_{1}^{\prime}$ and $B_{2}^{\prime}$, where

$$
B_{1}^{\prime}=\left\{\xi \in B^{\prime}|3| \xi \xi_{1}\left(\xi-\xi_{1}\right)\left|\leq \frac{1}{2}\right| \tau_{1}-\xi_{1}^{3} \mid\right\}
$$

and

$$
B_{2}^{\prime}=\left\{\xi \in B^{\prime}\left|\frac{1}{2}\right| \tau_{1}-\xi_{1}^{3}|\leq 3| \xi \xi_{1}\left(\xi-\xi_{1}\right)|\leq 3| \tau_{1}-\xi_{1}^{3} \mid\right\} .
$$

In $B_{1}^{\prime}$ we have that

$$
\frac{1}{2}\left|\tau_{1}-\xi_{1}^{3}\right| \leq\left|\tau_{1}-\xi_{1}^{3}+3 \xi \xi_{1}\left(\xi-\xi_{1}\right)\right|
$$

and

$$
|\xi| \leq \frac{1}{6}\left|\tau_{1}-\xi_{1}^{3}\right|
$$

Hence

$$
\begin{aligned}
I\left(B_{1}^{\prime}\right) & \leq \frac{c}{\left(1+\left|\tau_{1}-\xi_{1}^{3}\right|\right)^{1-\rho+b^{\prime}-b}}\left(\int_{|\xi| \leq\left|\tau_{1}-\xi_{1}^{3}\right|} \frac{|\xi|^{2(1-\rho)}}{(1+|\xi|)^{2 \rho}} d \xi\right)^{1 / 2} \\
& \leq c\left(1+\left|\tau_{1}-\xi_{1}^{3}\right|\right)^{1 / 2-\rho-b^{\prime}+b}
\end{aligned}
$$

which is bounded since, by hypothesis, $1 / 2-\rho-b^{\prime}+b \leq 0$. 
To bound $I\left(B_{2}^{\prime}\right)$ we split $B_{2}^{\prime}$ into three parts, $B_{2,1}^{\prime}, B_{2,2}^{\prime}$ and $B_{2,3}^{\prime}$.

First we have

$$
B_{2,1}^{\prime}=\left\{\xi \in B_{2}^{\prime}\left|\frac{1}{4}\right| \xi|\leq| \xi_{1}|\leq 100| \xi \mid\right\} .
$$

In this domain one has

$$
c\left(1+\left|\tau_{1}-\xi_{1}^{3}\right|\right) \leq\left|\xi_{1}\right|^{3} \sim|\xi|^{3} .
$$

Combining (2.35), the hypothesis $\rho>1 / 2$, a change of variable similar to that in (2.14)-(2.17), $\mu_{1}=\tau_{1}-\xi_{1}^{3}+3 \xi \xi_{1}\left(\xi-\xi_{1}\right)$, and (2.9) it follows that

$$
\begin{aligned}
& I\left(B_{2,1}^{\prime}\right) \leq c\left(1+\left|\tau_{1}-\xi_{1}^{3}\right|\right)^{\rho-b^{\prime}+(1-2 s) / 3} \times \\
& \left(\int_{\left|\mu_{1}\right| \leq 2\left|\tau_{1}-\xi_{1}^{3}\right|} \frac{d \xi}{\left(1+\left|\tau_{1}-\xi_{1}^{3}+3 \xi \xi_{1}\left(\xi-\xi_{1}\right)\right|\right)^{2(1-b)}}\right)^{1 / 2} \\
& \leq c\left(1+\left|\tau_{1}-\xi_{1}^{3}\right|\right)^{\rho / 3-b^{\prime}+1 / 3} \times \\
& \left(\frac{1}{|\xi|^{1 / 4}} \int_{\left|\mu_{1}\right|<2\left|\tau_{1}-\xi_{1}^{3}\right|} \frac{1}{\left(1+\left|\mu_{1}\right|\right)^{2(1-b)}} \frac{d \mu_{1}}{\left|\sqrt{4 \tau_{1}-\xi_{1}^{3}-4 \mu_{1}}\right|}\right)^{1 / 2} \\
& \leq c \frac{\left(1+\left|\tau_{1}-\xi_{1}^{3}\right|\right)^{\rho / 3-1 / 4+b-b^{\prime}}}{\left(1+\left|4 \tau_{1}-\xi_{1}^{3}\right|\right)^{1 / 4}},
\end{aligned}
$$

which is bounded since, by hypothesis, $\rho / 3-1 / 4+b-b^{\prime} \leq 0$.

Next we consider

$$
B_{2,2}^{\prime}=\left\{\xi \in B_{2}^{\prime}|1 \leq| \xi_{1}|\leq| \xi \mid / 4\right\}
$$

In this domain one has that

$$
\left|\xi_{1}-2 \xi\right| \sim|\xi| \sim\left|\xi-\xi_{1}\right|
$$

and

$$
c^{-1}\left(1+\left|\tau_{1}-\xi_{1}^{3}\right|\right) \leq\left|\xi \xi_{1}\left(\xi-\xi_{1}\right)\right| \sim|\xi|^{2}\left|\xi_{1}\right| \leq c|\xi|^{3} .
$$

Therefore (2.44), the change of variable $\mu_{1}=\lambda_{1}-\xi_{1}^{3}+3 \xi \xi_{1}\left(\xi-\xi_{1}\right)$ and $(2.11)$ give the inequalities

$$
\begin{aligned}
& I\left(B_{2,2}^{\prime}\right) \leq \frac{c}{\left(1+\left|\tau_{1}-\xi_{1}^{3}\right|\right)^{b^{\prime}}} \times \\
& \left(\int_{\left|\mu_{1}\right| \leq 3\left|\tau_{1}-\xi_{1}^{3}\right|} \frac{|\xi|^{2(1-2 \rho)}\left|\xi \xi_{1}\left(\xi-\xi_{1}\right)\right|^{2 \rho}}{\left|\xi_{1}\right|^{1 / 2}\left(1+\left|\tau_{1}-\xi_{1}^{3}\right|\right)^{1 / 2}\left(1+\left|\mu_{1}\right|\right)^{2(1-b)}} d \mu_{1}\right)^{1 / 2} \\
& \leq c\left(1+\left|\tau_{1}-\xi_{1}^{3}\right|\right)^{\theta},
\end{aligned}
$$


where

$$
\theta=-b^{\prime}+1 / 4+(1-2 \rho) / 3+\rho+b-1 / 2=\rho / 3-5 / 12+b-b^{\prime} \leq 0
$$

by using our hypothesis on $\rho, b$ and $b^{\prime}$. Hence $I\left(B_{2,2}^{\prime}\right)$ is bounded.

Finally we have

$$
B_{2,3}^{\prime}=\left\{\xi \in B_{2}^{\prime}|100| \xi|\leq| \xi_{1} \mid\right\}
$$

In this case one has that

$$
\left|\xi_{1}-2 \xi\right| \sim\left|\xi_{1}-\xi\right| \sim\left|\xi_{1}\right|, \quad\left|\tau_{1}-\xi_{1}^{3}\right|<<\left|\xi_{1}\right|^{3}
$$

and

$$
\left|4 \tau_{1}-\xi_{1}^{3}\right| \sim\left|\xi_{1}^{3}\right|
$$

Thus a familiar argument shows that

$$
\begin{aligned}
& I\left(B_{2,3}^{\prime}\right) \leq c\left(1+\left|\tau_{1}-\xi_{1}^{3}\right|\right)^{\rho-b^{\prime}} \times \\
& \left(\frac{1}{\left|\xi_{1}\right|^{1 / 2}} \int_{\left|\mu_{1}\right| \leq 3\left|\tau_{1}-\xi_{1}^{3}\right|} \frac{1}{\left(1+\left|\mu_{1}\right|\right)^{2(1-b)}} \frac{d \mu_{1}}{\mid \sqrt{4 \tau_{1}-\xi_{1}^{3}-4 \mu_{1} \mid}}\right)^{1 / 2} \\
& \leq c\left(1+\left|\tau_{1}-\xi_{1}^{3}\right|\right)^{\rho-5 / 6-b^{\prime}+b},
\end{aligned}
$$

which is bounded. This completes the proof of (2.28).

Proof of Theorem 2.2. First we consider the case $s=0$. Using Cauchy-Schwarz inequality, (2.12) and Fubini's theorem it follows that

$$
\begin{aligned}
& \left\|\frac{\xi}{\left(1+\left|\tau-\xi^{3}\right|\right)^{1-b}} \iint \frac{f\left(\xi_{1}, \tau_{1}\right)}{\left(1+\left|\tau_{1}-\xi_{1}^{3}\right|\right)^{b^{\prime}}} \frac{f\left(\xi-\xi_{1}, \tau-\tau_{1}\right) d \xi_{1} d \tau_{1}}{\left(1+\left|\tau-\tau_{1}-\left(\xi-\xi_{1}\right)^{3}\right|\right)^{b^{\prime}}}\right\|_{L_{\xi}^{2} L_{\tau}^{2}} \\
& \leq \| \frac{\xi}{\left(1+\left|\tau-\xi^{3}\right|\right)^{1-b}} \times \\
& \left(\iint \frac{d \xi_{1} d \tau_{1}}{\left(1+\left|\tau_{1}-\xi_{1}^{3}\right|\right)^{2 b^{\prime}}\left(1+\left|\tau-\tau_{1}-\left(\xi-\xi_{1}\right)^{3}\right|\right)^{2 b^{\prime}}}\right)^{1 / 2} \|_{L_{\xi}^{\infty} L_{\tau}^{\infty}} \\
& \left\|\left(\iint\left|f\left(\xi_{1}, \tau_{1}\right)\right|^{2}\left|f\left(\xi-\xi_{1}, \tau-\tau_{1}\right)\right|^{2} d \xi_{1} d \tau_{1}\right)^{1 / 2}\right\|_{L_{\xi}^{2} L_{\tau}^{2}} \\
& \leq c\|f\|_{L_{\xi}^{2} L_{\tau}^{2}}^{2}
\end{aligned}
$$

for any $b^{\prime} \in(1 / 2, b]$. Taking $b^{\prime}=b$ we obtain the result.

Next we turn to the case $\rho=-s \in(1 / 2,3 / 4)$. Note that if

$$
\text { either } \quad\left|\xi_{1}\right| \leq 1 \quad \text { or } \quad\left|\xi-\xi_{1}\right| \leq 1 \text {, }
$$


we have

$$
\left(1+\left|\xi_{1}\right|\right)^{\rho}\left(1+\left|\xi-\xi_{1}\right|\right)^{\rho} \leq c(1+|\xi|)^{\rho}
$$

which reduces the estimate to the case $\rho=0$. Thus, we can assume

$$
\left|\xi_{1}\right| \geq 1 \text { and }\left|\xi-\xi_{1}\right| \geq 1
$$

By symmetry we can also assume

$$
\left|\tau-\tau_{1}-\left(\xi-\xi_{1}\right)^{3}\right| \leq\left|\tau_{1}-\xi_{1}^{3}\right| .
$$

We split the region of integration in (2.7) into two parts

$$
\left|\tau_{1}-\xi_{1}^{3}\right| \leq\left|\tau-\xi^{3}\right| \text { and }\left|\tau-\xi^{3}\right| \leq\left|\tau_{1}-\xi_{1}^{3}\right| .
$$

For the first part we use Cauchy-Schwarz and (2.20) as in (2.51) to obtain the same bound. For the second part we use duality, Cauchy-Schwarz and (2.28) to conclude the proof.

We observe that Theorem 2.2 is equivalent to the following result.

Corollary 2.7. Given $s \in(-3 / 4,-1 / 2)$ there exists $b \in(1 / 2,1)$ such that for any $b^{\prime} \in(1 / 2, b]$ with $b-b^{\prime} \leq \min \{-s-1 / 2 ; 1 / 4+s / 3\}$

$$
\|B(F, F)\|_{X_{s, b-1}} \leq c\|F\|_{X_{s, b^{\prime}}}^{2} .
$$

Moreover (2.57) still holds for $s=0, b \in(1 / 2,3 / 4]$ and $b^{\prime} \in(1 / 2, b]$.

\section{Proof of Theorem 1.4}

We will denote by $\{W(t)\}_{-\infty}^{\infty}$ the unitary group describing the solution of the linear IVP associated to (1.1):

$$
\left\{\begin{array}{l}
\partial_{t} u+\partial_{x}^{3} u=0, \quad t, x \in \mathbb{R} \\
u(x, 0)=u_{0}(x)
\end{array}\right.
$$

where

$$
u(x, t)=W(t) u_{0}(x)=S_{t} * u_{0}(x)
$$

with $S_{t}(\cdot)$ defined by the oscillatory integral

$$
S_{t}(x)=c \int_{-\infty}^{\infty} e^{i x \xi} e^{i t \xi^{3}} d \xi .
$$

We will also use the notation

$$
\|f\|_{L_{x}^{p} L_{t}^{q}}=\left(\int_{-\infty}^{\infty}\left(\int_{-\infty}^{\infty}|f(x, t)|^{q} d t\right)^{p / q} d x\right)^{1 / q}
$$




$$
\widehat{J^{s} h}(\xi)=(1+|\xi|)^{s} \widehat{h}(\xi), \quad \widehat{D_{x}^{s} h}(\xi)=|\xi|^{s} \widehat{h}(\xi)
$$

and

$$
\widehat{\Lambda^{b}} g(\tau)=(1+|\tau|)^{b} \widehat{g}(\tau), \quad \widehat{D_{t}^{b}} g(\tau)=|\tau|^{b} \widehat{g}(\tau) .
$$

The relationship between the spaces $X_{s, b}$ in (1.6) and the group $\{W(t)\}_{-\infty}^{\infty}$ in (3.2) is described by the identity

$$
\|F\|_{X_{s, b}}=\left\|\Lambda^{b} J^{s} W(t) F\right\|_{L_{\xi}^{2} L_{\tau}^{2}} .
$$

Hence, if $F \in X_{s, b}$ then

$$
\begin{aligned}
F=F(x, t) & =\left((1+|\xi|)^{-s}\left(1+\left|\tau-\xi^{3}\right|\right)^{-b} \widehat{f}(\xi, \tau)\right)^{\vee} \\
& =\left((1+|\xi|)^{-s} \widehat{f}_{b}(\xi, \tau)\right)^{\vee}
\end{aligned}
$$

with $f \in L^{2}\left(\mathbb{R}^{2}\right)$. From the sharp version of the Kato smoothing effect [13] found in $[15]$, i.e.

$$
\left\|D_{x} W(t) v_{0}\right\|_{L_{x}^{\infty} L_{t}^{2}} \leq c\left\|v_{0}\right\|_{L^{2}}
$$

it follows that for $b>1 / 2$

$$
D_{x}^{\theta} f_{b} \in L_{x}^{2 /(1-\theta)} L_{t}^{2} \quad, \quad \text { for } \theta \in[0,1]
$$

In particular, this implies that for $s \in(-1,0)$

$$
F \in C_{\text {loc }}^{\alpha}\left(\mathbb{R}: L_{t}^{2}(\mathbb{R})\right) \quad \text { with } \quad \alpha \in(0,1+s) .
$$

Lemma 3.1. If $F \in X_{s, b}$ with $s \in(-1,0)$ and $b>1 / 2$, then

$$
F \in C_{\ell \circ \mathrm{oc}}^{\alpha}\left(\mathbb{R}: L_{t}^{2}(\mathbb{R})\right) \subseteq L_{x, \text { loc }}^{p}\left(\mathbb{R}: L_{t}^{2}(\mathbb{R})\right)
$$

for $1 \leq p \leq \infty$, and $\alpha \in(0,1+s)$.

Let $\psi \in C_{0}^{\infty}(\mathbb{R})$ with $\psi \equiv 1$ on $[-1,1]$ and supp $\psi \subseteq(-2,2)$. We recall the following results proven in [18].

Lemma 3.2. If $s \leq 0$ and $b \in(1 / 2,1]$, then for $\delta \in(0,1)$

$$
\begin{gathered}
\left\|\psi\left(\delta^{-1} t\right) W(t) v_{0}\right\|_{X_{s, b}} \leq c \delta^{(1-2 b) / 2}\left\|v_{0}\right\|_{H^{s}}, \\
\left\|\psi\left(\delta^{-1} t\right) F\right\|_{X_{s, b}} \leq c \delta^{(1-2 b) / 2}\|F\|_{X_{s, b}}, \\
\left\|\psi\left(\delta^{-1} t\right) \int_{0}^{t} W\left(t-t^{\prime}\right) F\left(t^{\prime}\right) d t^{\prime}\right\|_{X_{s, b}} \leq c \delta^{(1-2 b) / 2}\|F\|_{X_{s, b-1}}
\end{gathered}
$$


and

$$
\left\|\psi\left(\delta^{-1} t\right) \int_{0}^{t} W\left(t-t^{\prime}\right) F\left(t^{\prime}\right) d t^{\prime}\right\|_{H^{s}} \leq c \delta^{(1-2 b) / 2}\|F\|_{X_{s, b-1}} .
$$

Proof. For (3.13)-(3.15) see Lemmas 3.1-3.3 in [18]. The proof of (3.16) follows the same argument used in [18] to obtain (3.15).

Proof of Theorem 1.5. First we observe that if $u(\cdot)$ is a solution of the IVP (1.1), then for any $\lambda>0$

$$
u_{\lambda}(x, t)=\lambda^{2} u\left(\lambda x, \lambda^{3} t\right)
$$

also satisfies the KdV equation with data

$$
u_{\lambda}(x, 0)=\lambda^{2} u_{0}(\lambda x)
$$

Thus for $s \leq 0$

$$
\left\|u_{\lambda}(\cdot, 0)\right\|_{H^{s}}=0\left(\lambda^{3 / 2+s}\right) \quad \text { as } \quad \lambda \rightarrow 0
$$

In our case $s \in(-3 / 4,0]$, hence, without loss of generality, we can restrict ourselves to considering the IVP $(1.1)$ with data $u_{0}(x)$ satisfying

$$
\left\|u_{0}\right\|_{H^{s}}=r<<1
$$

i.e., $r$ arbitrary small; see (3.24) below.

For $u_{0} \in H^{s}(\mathbb{R}), s \in(-3 / 4,0]$, satisfying (3.20) we define the operator

$$
\Phi_{u_{0}}(\omega)=\Phi(\omega)=\psi(t) W(t) u_{0}-\frac{\psi(t)}{2} \int_{0}^{t} W\left(t-t^{\prime}\right) \psi^{2}\left(t^{\prime}\right) \partial_{x}\left(\omega^{2}\left(t^{\prime}\right)\right) d t^{\prime}
$$

We shall prove that $\Phi(\cdot)$ defines a contraction on

$$
\mathcal{B}(2 c r)=\left\{\omega \in X_{s, b} \quad \mid \quad\|\omega\|_{X_{s, b}} \leq 2 c r\right\} .
$$

First combining (3.13)-(3.15) and (1.9) (Theorem 1.1) it follows that for $\omega \in \mathcal{B}_{2 c r}$

$$
\begin{aligned}
\|\Phi(\omega)\|_{X_{s, b}} & \leq c r+c\left\|\partial_{x}(\psi(t) \omega(\cdot, t))^{2}\right\|_{X_{s, b-1}} \leq c r+c\|\psi \omega\|_{X_{s, b}}^{2} \\
& \leq c r+c\|\omega\|_{X_{s, b}}^{2} \leq c r+c(2 c r)^{2} \leq 2 c r
\end{aligned}
$$

for $r$ in (3.20) satisfying

$$
4 c^{2} r \leq 1
$$


Similarly using (3.14)-(3.15), (1.9) and (3.24) it follows that for $\omega, \widetilde{\omega} \in \mathcal{B}(2 c r)$

$$
\begin{aligned}
& \|\Phi(\omega)-\Phi(\widetilde{\omega})\|_{X_{s, b}} \\
& =\frac{1}{2}\left\|\psi(t) \int_{0}^{t} W\left(t-t^{\prime}\right) \psi^{2}\left(t^{\prime}\right) \partial_{x}\left(\omega^{2}-\widetilde{\omega}^{2}\right)\left(t^{\prime}\right) d t^{\prime}\right\|_{X_{s, b}} \\
& \leq c\|\omega+\widetilde{\omega}\|_{X_{s, b}}\|\omega-\widetilde{\omega}\|_{X_{s, b}} \leq 2 c^{2} r\|\omega-\widetilde{\omega}\|_{X_{s, b}} \\
& \leq \frac{1}{2}\|\omega-\widetilde{\omega}\|_{X_{s, b}} .
\end{aligned}
$$

Thus $\Phi(\cdot)$ is a contraction. Therefore there exists a unique $u \in \mathcal{B}(2 c r)$ such that

$$
u(t)=\psi(t)\left(W(t) u_{0}-\frac{1}{2} \int_{0}^{t} W\left(t-t^{\prime}\right) \partial_{x}\left(\psi\left(t^{\prime}\right) u\left(t^{\prime}\right)\right)^{2} d t^{\prime}\right) .
$$

Hence, in the time interval $[-1,1], u(\cdot)$ solves the integral equation associated to the IVP (1.1).

Now we turn to the proof of the persistence property, i.e.

$$
u \in C\left([-1,1]: H^{s}(\mathbb{R})\right)
$$

and the continuous dependence of the solution upon the data in the norm of the space in (3.27). Combining (3.7) and Hölder and Sobolev inequalities we obtain

$$
\begin{aligned}
& \left\|\psi\left(\rho^{-1} \cdot\right) u\right\|_{X_{s, 0}}=\left\|J^{s}\left(\psi\left(\rho^{-1} \cdot\right) u\right)\right\|_{L_{t}^{2} L_{x}^{2}} \\
& =\left\|W(t)\left(\psi\left(\rho^{-1} \cdot\right) J^{s} u\right)\right\|_{L_{t}^{2} L_{x}^{2}}=\left\|\psi\left(\rho^{-1} \cdot\right) W(t) J^{s} u\right\|_{L_{x}^{2} L_{t}^{2}} \\
& \leq c \rho^{1 / 4}\left\|\psi\left(\rho^{-1} \cdot\right) W(t) J^{s} u\right\|_{L_{x}^{2} L_{t}^{4}} \\
& \leq c \rho^{1 / 4}\left\|D_{t}^{1 / 4}\left(\psi\left(\rho^{-1} \cdot\right) W(t) J^{s} u\right)\right\|_{L_{x}^{2} L_{t}^{2}} \\
& =c \rho^{1 / 4}\left\|\psi\left(\rho^{-1} \cdot\right) u\right\|_{X_{s, 1 / 2}} \\
& \leq c \rho^{1 / 4}\left\|\psi\left(\rho^{-1} \cdot\right) u\right\|_{X_{s, b}} .
\end{aligned}
$$

Thus, by Hölder's inequality, (3.14) and (3.28), for $0<b \leq b^{\prime}$ we find that

$$
\begin{aligned}
& \left\|\psi\left(\rho^{-1} \cdot\right) u\right\|_{X_{s, b^{\prime}}} \leq\left\|\psi\left(\rho^{-1} \cdot\right) u\right\|_{X_{s, 0}}^{\left(b-b^{\prime}\right) / 2}\left\|\psi\left(\rho^{-1} \cdot\right) u\right\|_{X_{s, b}}^{b^{\prime} / b} \\
& \leq c \rho^{\left(b-b^{\prime}\right) / 8}\left\|\psi\left(\rho^{-1} \cdot\right) u\right\|_{X_{s, b}} \leq c \rho^{\left(b-b^{\prime}\right) / 8}\|u\|_{X_{s, b}}
\end{aligned}
$$

Using the integral equation (3.26), (2.57) with some fixed $b^{\prime}<b,(3.14),(3.16)$ 
and (3.29), for $0 \leq \tilde{t}<t \leq 1$ and $t-\tilde{t} \leq \Delta t$ it follows that

$$
\begin{aligned}
& \|u(t)-u(\tilde{t})\|_{H^{s}} \leq\|W(t-\tilde{t}) u(\tilde{t})-u(\tilde{t})\|_{H^{s}} \\
& +c\left\|\int_{\tilde{t}}^{t} W\left(t-t^{\prime}\right) \psi^{2}\left(\frac{t^{\prime}-\tilde{t}}{\Delta t}\right) \partial_{x}\left(u^{2}\left(t^{\prime}\right)\right) d t^{\prime}\right\| \\
& \leq\|W(t-\tilde{t}) u(\tilde{t})-u(\tilde{t})\|_{H^{s}}+c\left\|\partial_{x}\left(\psi^{2}\left(\frac{\cdot-\tilde{t}}{\Delta t}\right) u^{2}\right)\right\|_{X_{s, b-1}} \\
& \leq\|W(t-\tilde{t}) u(\tilde{t})-u(\tilde{t})\|_{H^{s}}+c\left\|\psi\left(\frac{\cdot-\tilde{t}}{\Delta t}\right) u\right\|_{X_{s, b^{\prime}}}^{2} \\
& \leq\|W(t-\tilde{t}) u(\tilde{t})-u(\tilde{t})\|_{H^{s}}+c(\Delta t)^{\left(b-b^{\prime}\right) / 4}\|u\|_{X_{s, b}}^{2}=o(1)
\end{aligned}
$$

as $\Delta t \rightarrow 0$, which yields the persistence property. The proof of the continuous dependence of the solution upon the data in the $L^{\infty}\left([0,1]: H^{s}(\mathbb{R})\right)$-norm follows a similar argument, therefore it will be omitted.

Finally we explain how to extend the uniqueness result in $\mathcal{B}(2 c r)$ in $(3.22)$ to the whole $X_{s, b}$. For any $\delta \in(0,1)$ we define

$$
\mathcal{B}\left(2 c \delta^{(1-2 b) / 2} r\right)=\left\{\omega \in X_{s, b} \mid\|\omega\|_{X_{s, b}} \leq 2 c \delta^{(1-2 b) / 2} r\right\}
$$

and

$$
\Phi_{\delta, u_{0}}(\omega)=\Phi_{\delta}(\omega)=\psi\left(\delta^{-1} t\right) W(t) u_{0}-\frac{\psi\left(\delta^{-1} t\right)}{2} \int_{0}^{t} W\left(t-t^{\prime}\right) \psi^{2}\left(t^{\prime}\right) \partial_{x}\left(\omega^{2}\left(t^{\prime}\right)\right) d t^{\prime} .
$$

Combining (2.57) with $b^{\prime} \in\left(\frac{1}{2}, b\right)$ such that

$$
b-b^{\prime}=\min \{-s-1 / 2 ; 1 / 4+s / 3\}
$$

with the arguments in (3.23) and (3.29) one has

$$
\left\|\Phi_{\delta}(\omega)\right\|_{X_{s, b}} \leq c \delta^{(1-2 b) / 2} r+4 c^{3} \delta^{3(1-2 b) / 2} \delta^{\left(b-b^{\prime}\right) / 4} r^{2},
$$

and

$$
\left\|\Phi_{\delta}(\omega)-\Phi_{\delta}(\widetilde{\omega})\right\|_{X_{s, b}} \leq 2 c^{3} \delta^{(1-2 b)} \delta^{\left(b-b^{\prime}\right) / 4} r\|\omega-\widetilde{\omega}\|_{X_{s, b}} .
$$

By (3.33) it follows that

$$
1-2 b+\left(b-b^{\prime}\right) / 4<0,
$$

therefore for any $\delta \in(0,1)$

$$
\Phi_{\delta}\left(\mathcal{B}\left(2 c \delta^{(1-2 b) / 2} r\right)\right) \subseteq \mathcal{B}\left(2 c \delta^{(1-2 b) / 2} r\right)
$$

is a contraction, which completes the proof. 


\section{Proof of Theorems 1.3 And 1.7}

Proof of Theorem 1.3. Using the notation in (2.1)-(2.5), one sees that (1.9) is equivalent to

$$
\begin{aligned}
& \| \frac{\xi}{\left(1+\left|\tau-\xi^{3}\right|\right)^{1-b}(1+|\xi|)^{\rho}} \times \\
& \quad \iint \frac{f\left(\xi_{1}, \tau_{1}\right)\left(1+\left|\xi_{1}\right|\right)^{\rho}}{\left(1+\left|\tau_{1}-\xi_{1}^{3}\right|\right)^{b}} \frac{f\left(\xi-\xi_{1}, \tau-\tau_{1}\right)\left(1+\left|\xi-\xi_{1}\right|\right)^{\rho} d \xi_{1} d \tau_{1}}{\left(1+\left|\tau-\tau_{1}-\left(\xi-\xi_{1}\right)^{3}\right|\right)^{b}} \|_{L_{\xi}^{2} L_{\tau}^{2}} \\
& \quad \leq c\|f\|_{L_{\xi}^{2} L_{\tau}^{2}}^{2} .
\end{aligned}
$$

First we shall see that if $\rho=-s>3 / 4$, then $b \leq 1 / 2$.

Fix $N \in \mathbb{Z}^{+}$and take

$$
f(\xi, \tau)=\chi_{A}(\xi, \tau)+\chi_{-A}(\xi, \tau),
$$

where $\chi_{A}(\cdot)$ represents the characteristic function of the set $A$ with

$$
A=\left\{(\xi, \tau) \in \mathbb{R}^{2}\left|N \leq \xi \leq N+\frac{1}{\sqrt{N}},\right| \tau-\xi^{3} \mid \leq 1\right\}
$$

and

$$
-A=\left\{(\xi, \tau) \in \mathbb{R}^{2} \mid-(\xi, \tau) \in A\right\}
$$

Clearly

$$
\|f\|_{L_{\xi}^{2} L_{\tau}^{2}} \leq c N^{-\frac{1}{4}}
$$

On the other hand, $A$ contains a rectangle with $\left(N, N^{3}\right)$ as a vertex, with dimensions $10^{-2} N^{-2} \times N^{3 / 2}$ and longest side pointing in the $\left(1,3 N^{2}\right)$ direction.

Therefore

$$
|(f * f)(\xi, \tau)| \geq \frac{c}{\sqrt{N}} \chi_{\mathcal{R}}(\xi, \tau)
$$

where $\mathcal{R}$ is the rectangle centered at the origin of dimensions $c N^{-2} \times N^{\frac{3}{2}}$ and longest side pointing in the $\left(1,3 N^{2}\right)$ direction. Hence (4.1) implies that

$$
N^{2 \rho} N^{-\frac{1}{2}} N^{\frac{3}{2}(b-1)} N^{-\frac{1}{2}} N^{\frac{1}{4}} \leq c N^{-\frac{1}{2}},
$$

i.e.

$$
b+4 \rho \leq \frac{3}{2}
$$

as desired. 
To complete the proof we just need to show that if $\rho=-s>3 / 4$ then $b>1 / 2$. By polarization and duality (4.1) is equivalent to the following inequality:

$$
\begin{aligned}
& \| \frac{\left(1+\left|\xi_{1}\right|\right)^{\rho}}{\left(1+\left|\tau_{1}-\xi_{1}^{3}\right|\right)^{b}} \times \\
& \iint \frac{|\xi|}{(1+|\xi|)^{s}} \frac{g(\xi, \tau)}{\left(1+\left|\tau-\xi^{3}\right|\right)^{1-b}} \frac{h\left(\xi-\xi_{1}, \tau-\tau_{1}\right)\left(1+\left|\xi-\xi_{1}\right|\right)^{s}}{\left(1+\left|\tau-\tau_{1}-\left(\xi-\xi_{1}\right)^{3}\right|\right)^{b}} d \xi d \tau \|_{L_{\xi_{1}}^{2} L_{\tau_{1}}^{2}} \\
& \leq c\|g\|_{L_{\xi}^{2} L_{\tau}^{2}}\|h\|_{L_{\xi}^{2} L_{\tau}^{2}} .
\end{aligned}
$$

We take

$$
g(\xi, \tau)=\chi_{A}(\xi, \tau),(A \text { defined in }(4.3))
$$

and

$$
h(\xi, \tau)=\chi_{B}(\xi, \tau)
$$

where

$$
B=\left\{(\xi, \tau) \in \mathbb{R}^{2}|-N+1 / 2 \sqrt{N} \leq \xi \leq-N+3 / 4 \sqrt{N} ;| \tau-\xi^{3} \mid<1\right\}
$$

Estimating the first norm in (4.9) in the domain

$$
\left|4 \tau_{1}-\xi_{1}^{3}\right| \leq \frac{1}{4} \quad \text { and } \quad \xi_{1} \in\left[N-\frac{1}{20} \frac{1}{\sqrt{N}}, N-\frac{1}{10} \frac{1}{\sqrt{N}}\right]
$$

from (4.9) we see that

$$
\frac{N^{\rho+1 / 4}}{N^{3 b}} \leq N^{-1 / 2}
$$

i.e. if $\rho=-s>3 / 4$

$$
\frac{3}{2}<\rho+\frac{3}{4} \leq 3 b
$$

Proof of Theorem 1.7. We consider the case $b=1 / 2$, and remark that a similar argument to that used in the previous proof shows that this assumption does not imply a loss of generality.

First we take $F(\cdot, \cdot)$ such that

$$
\widehat{F}(\xi, \tau)=\chi_{A}(\xi, \tau)+\chi_{-A}(\xi, \tau),
$$

where $A$ was defined in (4.3). Thus

$$
|(\widehat{F} * \widehat{F} * \widehat{F})(\xi, \tau)| \geq \frac{c}{\sqrt{N}}\left|\left(\chi_{A} * \chi_{\mathcal{R}}\right)(\xi, \tau)\right|,
$$


where $\mathcal{R}$ represents the rectangle centered at the origin of dimension $10^{-2} N^{-2} \times$ $N^{3 / 2}$ and with its longest side pointing in the $\left(1,3 N^{2}\right)$ direction.

Then

$$
|(\widehat{F} * \widehat{F} * \widehat{F})(\xi, \tau)| \geq \frac{c}{N} \chi_{\mathcal{R}_{0}}(\xi, \tau),
$$

where $\mathcal{R}_{0}$ is a rectangle like $\mathcal{R}$, of the same dimensions and direction, but with $\left(N, N^{3}\right)$ as one of its vertices. Hence

$$
\|\widetilde{B}(F, F, F)\|_{X_{s, b-1}} \geq c N^{s-1 / 4} .
$$

Since

$$
\|F\|_{X_{s, b}} \leq c N^{s-1 / 4}
$$

(1.18) implies that $s \geq 1 / 4$, which completes the proof.

\section{Proof of Theorem 1.2}

First we rewrite the inequality (1.10). Defining

$$
\rho=-s \in[0,1 / 2)
$$

by (1.7) it follows that if $F \in Y_{s, b}=Y_{-\rho, b}$, then

$$
f(n, \tau)=\left(1+\left|\tau-n^{3}\right|\right)^{b}|n|^{-\rho} \widehat{F}(n, \tau) \in L_{\tau}^{2}\left(\mathbb{R}: \ell_{n}^{2}(\mathbb{T})\right)
$$

and

$$
\|f\|_{L_{\tau}^{2} \ell_{n}^{2}}=\|F\|_{Y_{-\rho, b}}
$$

Since

$$
\left.\widehat{\partial_{x}\left(F^{2}\right.}\right)(n, \tau)=c n(\widehat{F} * \widehat{F})(n, \tau)
$$

we can express (1.10) in terms of $f$ as

$$
\begin{aligned}
& \left.\|B(F, F)\|_{Y_{s, b-1,0}}=\|\left(1+\left|\tau-n^{3}\right|\right)^{b-1}|n|^{-\rho} \widehat{\partial_{x}\left(F^{2}\right.}\right)(n, \tau) \|_{L_{\tau}^{2} \ell_{n}^{2}} \\
& =c\left\|\left(1+\left|\tau-n^{3}\right|\right)^{b-1}|n|^{-\rho} n(\widehat{F} * \widehat{F})(n, \tau)\right\|_{L_{\tau}^{2} \ell_{n}^{2}} \\
& =\left(\sum_{n \neq 0} \int_{-\infty}^{\infty} \frac{|n|^{2}}{\left(1+\left|\tau-n^{3}\right|\right)^{2(1-b)}|n|^{2 \rho}} \times\right. \\
& \mid \sum_{\substack{n_{1} \neq n \\
n_{1} \neq 0}} \int_{-\infty}^{\infty} \frac{\left|n_{1}\left(n-n_{1}\right)\right|^{\rho}}{\left(1+\left|\tau_{1}-n_{1}^{3}\right|\right)^{1 / 2}} \times \\
& \left.\left.\frac{f\left(n_{1}, \tau_{1}\right)}{\left(1+\left|\tau_{1}-n_{1}^{3}\right|\right)^{1 / 2}} \frac{f\left(n-n_{1}, \tau-\tau_{1}\right) d \tau_{1}}{\left(1+\left|\tau-\tau_{1}-\left(n-n_{1}\right)^{3}\right|\right)^{1 / 2}}\right|^{2}\right)^{1 / 2} \\
& =\|\mathcal{B}(f, f)(n, \tau)\|_{L_{\tau}^{2} \ell_{n}^{2}} \leq c\left(\sum_{n \neq 0}^{\infty} \int_{-\infty}^{\infty}|f(n, \tau)|^{2} d \tau\right)=c\|f\|_{L_{\tau}^{2} \ell_{n}^{2}}^{2} .
\end{aligned}
$$

As in the continuous case we first need some calculus results. 
Lemma 5.1. There exists $c>0$ such that for any $n \neq 0$ and any $\tau \in \mathbb{R}$

$$
\sum_{\substack{n_{1} \neq 0 \\ n_{1} \neq n}} \frac{\log \left(2+\left|\tau-n n_{1}\left(n-n_{1}\right)\right|\right)}{1+\left|\tau-n n_{1}\left(n-n_{1}\right)\right|}<c .
$$

Proof. Since $n \neq 0$ it suffices to show that

$$
\left\|\sum_{\substack{n_{1} \neq 0 \\ n_{1} \neq n}} \frac{1}{\left(1+\left|\mu-n_{1}\left(n-n_{1}\right)\right|\right)^{3 / 4}}\right\|_{L_{\mu}^{\infty} \ell_{n}^{\infty}}<c .
$$

Let $\alpha, \beta$ be the roots of the equation

$$
\mu-n_{1}\left(n-n_{1}\right)=0
$$

i.e.

$$
\mu-n_{1}\left(n-n_{1}\right)=\left(n_{1}-\alpha\right)\left(n_{1}-\beta\right), \text { with } \alpha=\alpha(n, \mu), \beta=\beta(n, \mu) .
$$

We shall prove that

$$
\left\|\sum_{n_{1}} \frac{1}{\left(1+\left|\left(n_{1}-\alpha\right)\left(n_{1}-\beta\right)\right|\right)^{3 / 4}}\right\|_{L_{\alpha}^{\infty} L_{\beta}^{\infty}}<c
$$

which implies (5.7).

Notice that for a fixed $(\alpha, \beta) \in \mathbb{R}^{2}$ there exist at most $8 n_{1}$ 's such that

$$
\left|n_{1}-\alpha\right| \leq 2 \text { or }\left|n_{1}-\beta\right| \leq 2
$$

For the remaining $n_{1}$ 's one has that

$$
1+\left|n_{1}-\alpha\right|\left|n_{1}-\beta\right| \geq \frac{1}{2}\left(1+\left|n_{1}-\alpha\right|\right)\left(1+\left|n_{1}-\beta\right|\right),
$$

hence

$$
\begin{aligned}
& \sum_{n_{1}} \frac{1}{\left(1+\left|\left(n_{1}-\alpha\right)\left(n_{1}-\beta\right)\right|\right)^{3 / 4}} \leq 8+\sum_{\substack{\left|n_{1}-\alpha\right| \geq 2 \\
\left|n_{1}-\beta\right| \geq 2}} \frac{1}{\left(1+\left|\left(n_{1}-\alpha\right)\left(n_{1}-\beta\right)\right|\right)^{3 / 4}} \\
& \leq 8+\left(\sum_{\left|n_{1}-\alpha\right| \geq 2}^{1 / 2} \frac{1}{\left(1+\left|n_{1}-\alpha\right|\right)^{3 / 2}}\right)^{1 / 2}\left(\sum_{\left|n_{1}-\beta\right| \geq 2} \frac{1}{\left(1+\left|n_{1}-\beta\right|\right)^{3 / 2}}\right)^{<}<c,
\end{aligned}
$$

which yields the lemma. 
Lemma 5.2. If $\rho=-s \in[0,1 / 2]$, then

$$
\begin{aligned}
& \frac{n}{\left(1+\left|\tau-n^{3}\right|\right)^{1 / 2}} \frac{1}{|n|^{\rho}} \times \\
& \left(\sum_{n_{1} \in A} \int_{A} \frac{\left|n_{1}\left(n-n_{1}\right)\right|^{2 \rho}}{\left(1+\left|\tau_{1}-n_{1}^{3}\right|\right)\left(1+\left|\tau-\tau_{1}-\left(n-n_{1}\right)^{3}\right|\right)} d \tau_{1}\right)^{1 / 2}<c,
\end{aligned}
$$

where

$$
\begin{aligned}
A=A(n, \tau) & =\left\{\left(n_{1}, \tau_{1}\right) \in \mathbb{Z} \times \mathbb{R} \mid n_{1} \neq 0, n_{1} \neq n,\right. \\
& \left.\left|\tau-\tau_{1}-\left(n-n_{1}\right)^{3}\right| \leq\left|\tau_{1}-n_{1}^{3}\right| \leq|\tau-n|\right\} .
\end{aligned}
$$

Proof. It suffices to prove (5.13) for $s=0$ and $s=-1 / 2$. The general case follows by the Three Lines Theorem. As in [4] we shall use the identity

$$
\tau-n^{3}-\left(\tau_{1}-n_{1}^{3}\right)-\left(\tau-\tau_{1}-\left(n-n_{1}\right)^{3}\right)=3 n n_{1}\left(n-n_{1}\right),
$$

which implies that

$$
\max \left\{\left|\tau-n^{3}\right| ;\left|\tau_{1}-n_{1}^{3}\right| ;\left|\tau-\tau_{1}-\left(n-n_{1}\right)^{3}\right|\right\} \geq\left|n n_{1}\left(n-n_{1}\right)\right|
$$

Case $s=0$. Combining (5.16) and the definition of $A$ it follows that

$$
\left|\tau-n^{3}\right| \geq\left|n n_{1}\left(n-n_{1}\right)\right| \geq n^{2} / 2 .
$$

Hence we only have to show that

$$
\sum_{n_{1} \in A} \int_{-\infty}^{\infty} \frac{d \tau_{1}}{\left(1+\left|\tau_{1}-n_{1}^{3}\right|\right)\left(1+\left|\tau-\tau_{1}-\left(n-n_{1}\right)^{3}\right|\right)}<c
$$

Changing variable

$$
\theta=\tau_{1}-n_{1}^{3}
$$

and using the notation

$$
a=\tau-n^{3}+3 n n_{1}\left(n-n_{1}\right)
$$

we find that

$$
\begin{aligned}
& \int_{-\infty}^{\infty} \frac{d \tau_{1}}{\left(1+\left|\tau_{1}-n_{1}^{3}\right|\right)\left(1+\left|\tau-\tau_{1}-\left(n-n_{1}\right)^{3}\right|\right)} \\
= & \int_{-\infty}^{\infty} \frac{d \theta}{(1+|\theta|)(1+|\theta-a|)} \leq \frac{c \log (2+|a|)}{1+|a|} .
\end{aligned}
$$

Inserting (5.21) into (5.18) and using Lemma 5.1 we complete the proof. 
Case $\rho=-s=1 / 2$. In this case we have that in $A$

$$
\left(1+\left|\tau-n^{3}\right|\right)^{1 / 2} \geq|n|^{1 / 2}\left|n_{1}\left(n-n_{1}\right)\right|^{1 / 2} .
$$

Therefore the proof reduces to the previous case.

Lemma 5.3. If $\rho=-s \in[0,1 / 2]$, then

$$
\begin{gathered}
\frac{1}{\left(1+\left|\tau_{1}-n_{1}^{3}\right|\right)^{1 / 2}} \times \\
\left(\sum_{n \in D} \int_{D} \frac{|n|^{2(1-2 \rho)}\left|n n_{1}\left(n-n_{1}\right)\right|^{2 \rho} d \tau}{\left(1+\left|\tau-n^{3}\right|\right)\left(1+\left|\tau-\tau_{1}-\left(n-n_{1}\right)^{3}\right|\right)}\right)^{1 / 2}<c,
\end{gathered}
$$

where

$$
\begin{aligned}
& D=D\left(n_{1}, \tau_{1}\right)=\left\{(n, \tau) \in \mathbb{Z} \times \mathbb{R} \mid n \neq n_{1},\right. \\
& \left.\quad\left|\tau-n^{3}\right| \leq\left|\tau_{1}-n_{1}^{3}\right|,\left|\tau-\tau_{1}-\left(n-n_{1}\right)^{3}\right| \leq\left|\tau_{1}-n_{1}^{3}\right|\right\} .
\end{aligned}
$$

Proof. As in the previous case it suffices to consider the cases $s=0$ and $s=-1 / 2$.

Case $s=0$. From the definition of $D$ and the relations (5.15)-(5.16) it follows that

$$
\left|\tau_{1}-n_{1}^{3}\right| \geq n^{2} / 2
$$

Hence the proof reduces to that in Lemma 5.2, case $s=0$.

Case $\rho=-s=1 / 2$. Since

$$
\left(1+\left|\tau_{1}-n_{1}^{3}\right|\right)^{1 / 2} \geq|n|^{1 / 2}\left|n_{1}\left(n-n_{1}\right)\right|^{1 / 2}
$$

the numerator in the integrand in (5.23) cancels the expression outside the parentheses. Hence the proof follows the argument in the previous case.

Proof of Theorem 1.2. Using (5.2)-(5.3), and the Cauchy-Schwarz inequality we 
write

$$
\begin{aligned}
& \|B(F, F)\|_{Y_{s, b-1}}=\|\mathcal{B}(f, f)\|_{L_{\tau}^{2} \ell_{n}^{2}} \\
& =\left(\sum_{n \neq 0} \int_{-\infty}^{\infty} \frac{n^{2}}{\left(1+\left|\tau-n^{3}\right|\right)} \frac{1}{|n|^{2 \rho}} \times\right. \\
& \left.\left|\sum_{\substack{n_{1} \neq n \\
n_{1} \neq 0}} \int_{-\infty}^{\infty} \frac{\left|n_{1}\left(n-n_{1}\right)\right|^{\rho} f\left(n_{1}, \tau_{1}\right) f\left(n-n_{1}, \tau-\tau_{1}\right)}{\left(1+\left|\tau_{1}-n_{1}^{2}\right|\right)^{1 / 2}\left(1+\left|\tau-\tau_{1}-\left(n-n_{1}\right)^{3}\right|\right)^{1 / 2}} d \tau_{1}\right|^{2} d \tau\right)^{1 / 2} \\
& \leq\left(\sum_{n \neq 0} \int_{-\infty}^{\infty} \frac{n^{2}}{\left(1+\left|\tau-n^{3}\right|\right)} \frac{1}{|n|^{2 \rho}} \times\right. \\
& \left(\sum_{\substack{n_{1} \neq n \\
n_{1} \neq 0}} \int_{-\infty}^{\infty} \frac{\left|n_{1}\left(n-n_{1}\right)\right|^{2 \rho}}{\left(1+\left|\tau_{1}-n_{1}^{3}\right|\right)\left(1+\left|\tau-\tau_{1}-\left(n-n_{1}\right)^{3}\right|\right.} d \tau_{1}\right) \\
& \left.\left(\sum_{\substack{n_{1} \neq n \\
n_{1} \neq 0}} \int_{-\infty}^{\infty}\left|f\left(n_{1}, \tau_{1}\right) f\left(n-n_{1}, \tau-\tau_{1}\right)\right|^{2} d \tau_{1}\right) d \tau\right)^{1 / 2} \\
& \leq \| \frac{n}{\left(1+\left|\tau-n^{3}\right|\right)^{1 / 2}} \frac{1}{|n|^{\rho}} \times \\
& \left(\sum_{\substack{n_{1} \neq 0 \\
n_{1} \neq n}} \int_{-\infty}^{\infty} \frac{\left|n_{1}\left(n-n_{1}\right)\right|^{2 \rho}}{\left(1+\left|\tau_{1}-n_{1}^{3}\right|\right)\left(1+\left|\tau-\tau_{1}-\left(n-n_{1}\right)^{3}\right|\right)} d \tau_{1}\right)^{1 / 2} \|_{L_{\tau}^{\infty} \ell_{n}^{\infty}} \\
& \left(\sum_{n \neq 0} \sum_{\substack{n_{1} \neq n \\
n_{1} \neq 0}} \iint\left|f\left(n_{1}, \tau_{1}\right)\right|^{2}\left|f\left(n-n_{1}, \tau-\tau_{1}\right)\right|^{2} d \tau_{1} d \tau\right)^{1 / 2} \\
& =\Psi\|f\|_{L_{\tau}^{2} \ell_{n}^{2}}^{2} .
\end{aligned}
$$

To complete the proof we just need to show that $\Psi$, defined above, is bounded. But this follows by combining Lemmas 5.2 and 5.3, using duality and symmetry arguments.

\section{Proof of Theorems $1.4,1.8$}

We recall the notation introduced in (5.5)

$$
\begin{aligned}
& \mathcal{B}_{\rho, b}(f, f)(n, \tau)=\frac{n}{\left(1+\left|\tau-n^{3}\right|\right)^{1-b}} \frac{1}{|n|^{\rho}} \times \\
& \sum_{\substack{n_{1} \neq 0 \\
n_{1} \neq n}} \int_{-\infty}^{\infty} \frac{\left|n_{1}\left(n-n_{1}\right)\right|^{\rho} f\left(n_{1}, \tau_{1}\right) f\left(n-n_{1}, \tau-\tau_{1}\right)}{\left(1+\left|\tau_{1}-n_{1}^{3}\right|\right)^{b}\left(1+\left|\tau-\tau_{1}-\left(n-n_{1}\right)^{3}\right|\right)^{b}} d \tau_{1} .
\end{aligned}
$$

First we shall see that the condition

$$
b=\frac{1}{2}
$$

is necessary for the boundedness of $\mathcal{B}_{\rho, b}(f, f)$. 
Lemma 6.1. If there exists $\rho \in \mathbb{R}$ such that for any $f \in L_{\tau}^{2} \ell_{n}^{2}$

$$
\left\|\mathcal{B}_{\rho, b}(f, f)\right\|_{L_{\tau}^{2} \ell_{n}^{2}} \leq c\|f\|_{L_{\tau}^{2} \ell_{n}^{2}}^{2},
$$

then

$$
b=\frac{1}{2}
$$

\section{Proof. Choose}

$$
f(n, \tau)=a_{n} \chi_{1 / 2}\left(\tau-n^{3}\right)
$$

where

$$
a_{n}= \begin{cases}1, & n=1, \quad N-1, \\ 0, & \text { elsewhere }\end{cases}
$$

and

$$
\chi_{\theta}(x)= \begin{cases}1, & |x|<\theta \\ 0, & \text { elsewhere }\end{cases}
$$

We evaluate $\mathcal{B}_{\rho, b}(f, f)(n, \tau)$ at $n=N$ and bound it below by

$$
\frac{N}{\left(1+\left|\tau-N^{3}\right|\right)^{1-b}}
$$

for those $\tau$ 's for which there is a $\tau_{1}$ interval of size larger than a fixed positive constant such that

$$
\left|\tau_{1}-1\right| \leq \frac{1}{2} \text { and }\left|\tau-\tau_{1}-(N-1)^{3}\right| \leq \frac{1}{2} .
$$

Assuming that

$$
\left|\tau_{1}-1\right| \leq \frac{1}{4} \text { and }\left|\tau-\tau_{1}-N^{3}+1+3 N(N-1)\right|<\frac{1}{2}
$$

we have that (6.9) holds and

$$
\left|\tau-N^{3}\right| \sim N^{2}
$$

Hence, (6.3) implies that

$$
\frac{N}{N^{2(1-b)}} \leq c \quad \text { for any } N \in \mathbb{Z}^{+}
$$

and consequently

$$
b \leq 1 / 2 .
$$


To see that $b \geq 1 / 2$ we note that polarization and duality show that if $(6.3)$ holds, then a similar inequality must hold for $\widetilde{\mathcal{B}}_{\rho, b}(\cdot, \cdot)$, where

$$
\begin{aligned}
& \widetilde{\mathcal{B}}_{\rho, b}(f, f)\left(n_{1}, \tau_{1}\right)=\frac{1}{\left(1+\left|\tau_{1}-n_{1}^{3}\right|\right)^{b}} \times \\
& \sum_{\substack{n \neq 0 \\
n \neq n_{1}}} \int_{-\infty}^{\infty} \frac{|n| f(n, \tau)}{\left(1+\left|\tau-n^{3}\right|\right)^{1-b}|n|^{\rho}} \frac{\left|n_{1}\left(n-n_{1}\right)\right|^{\rho} f\left(n-n_{1}, \tau-\tau_{1}\right)}{\left(1+\left|\tau-\tau_{1}-\left(n-n_{1}\right)^{3}\right|\right)^{b}} d \tau,
\end{aligned}
$$

i.e., (6.3) is equivalent to

$$
\left\|\widetilde{\mathcal{B}}_{\rho, b}(f, f)\right\|_{L_{\tau_{1}}^{2} \ell_{n_{1}}^{2}} \leq c\|f\|_{L_{\tau}^{2} \ell_{n}^{2}}^{2} .
$$

We choose

$$
f(n, \tau)=b_{n} \chi_{1 / 2}\left(\tau-n_{3}\right)
$$

where

$$
b_{n}= \begin{cases}1, & n=N-1, N \\ 0, & \text { elsewhere }\end{cases}
$$

and we evaluate $\widetilde{\mathcal{B}}_{\rho, b}(f, f)\left(n_{1}, \tau_{1}\right)$ at $n_{1}=1$ and bound it below by

$$
\frac{N}{\left(1+\left|\tau_{1}-1\right|\right)^{b}}
$$

for those $\tau_{1}$ 's for which there is a $\tau$ interval of size larger than a fixed positive constant such that

$$
\left|\tau-N^{3}\right| \leq \frac{1}{2} \quad \text { and } \quad\left|\tau-\tau_{1}-(N-1)^{3}\right| \leq \frac{1}{2}
$$

Clearly (6.19) holds in an interval of $\tau_{1}$ 's of length larger than $\frac{1}{2}$ in which

$$
\left|\tau_{1}-1\right| \sim N^{2}
$$

Hence (6.15) implies that

$$
\frac{N}{N^{2 b}} \leq c \quad \text { for any } N \in \mathbb{Z}^{+}
$$

and consequently

$$
b \geq \frac{1}{2}
$$

which completes the proof.

Proof of Theorem 1.4. By Lemma 6.1, it suffices to see that (6.3) with $b=1 / 2$ fails for $\rho=-s>1 / 2$. 
We choose

$$
f(n, \tau)=a_{n} \chi_{1}\left(\tau-n^{3}\right)
$$

where

$$
a_{n}= \begin{cases}1, & n=N / 2 \\ 0, & \text { elsewhere }\end{cases}
$$

We evaluate $\mathcal{B}_{\rho, 1 / 2}(f, f)(n, \tau)$ at $n=N$ and bound it below by

$$
\frac{N}{\left(1+\left|\tau-N^{3}\right|\right)^{1 / 2}} \frac{1}{N^{s}} N^{2 s}
$$

for those $\tau$ 's for which in a unit size interval of $\tau_{1}$ 's we have

$$
\left|\tau_{1}-(N / 2)^{3}\right| \leq 1 \quad \text { and } \quad\left|\tau-\tau_{1}-(N / 2)^{3}\right| \leq 1
$$

Assuming that

$$
\left|\tau_{1}-(N / 2)^{3}\right| \leq \frac{1}{2} \quad \text { and } \quad\left|\tau-2(N / 2)^{3}\right| \leq 1
$$

it follows that (6.26) is satisfied. Moreover

$$
\left|\tau-N^{3} / 4\right| \leq \frac{1}{2} \quad \text { implies } \quad\left|\tau-N^{3}\right| \sim N^{3} .
$$

Hence

$$
\frac{N}{N^{3 / 2}} N^{\rho} \leq c \quad \text { for any } N \in \mathbb{Z}^{+}
$$

Therefore

$$
\rho \leq \frac{1}{2}
$$

Proof of Theorem 1.8. Using the notation in (6.1) we define

$$
\begin{aligned}
& \mathcal{B}_{3}(f, f, f)(n, \tau)=\frac{n}{\left(1+\left|\tau-n^{3}\right|\right)^{1-b}} \frac{1}{(1+|n|)^{\rho}} \times \\
& \sum_{n_{2} \neq n} \sum_{\substack{n_{1} \neq n_{2} \\
n_{1} \neq 0}} \int_{-\infty}^{\infty} \int_{-\infty}^{\infty} \frac{f\left(n_{1}, \tau_{1}\right)\left(1+\left|n_{1}\right|\right)^{\rho}}{\left(1+\left|\tau_{1}-n_{1}^{3}\right|\right)^{b}} \frac{f\left(n_{2}-n_{1}, \tau_{2}-\tau_{1}\right)\left(1+\left|n_{2}-n_{1}\right|\right)^{\rho}}{\left(1+\left|\tau_{2}-\tau_{1}-\left(n_{2}-n_{1}\right)^{3}\right|\right)^{b}} \times \\
& \frac{f\left(n-n_{2}, \tau-\tau_{2}\right)\left(1+\left|n-n_{2}\right|\right)^{\rho}}{\left(1+\left|\tau-\tau_{1}-\left(n-n_{2}\right)^{3}\right|\right)^{b}} d \tau_{1} d \tau_{2} .
\end{aligned}
$$


Thus to obtain (1.19) it suffices to show that

$$
\left\|\mathcal{B}_{3}(f, g, h)\right\|_{L_{\tau}^{2} \ell_{n}^{2}} \leq c\|f\|_{L_{\tau}^{2} \ell_{n}^{2}}\|g\|_{L_{\tau}^{2} \ell_{n}^{2}}\|h\|_{L_{\tau}^{2} \ell_{n}^{2}}
$$

fails for any $\rho=-s>1 / 2$.

An argument similar to that used in the proof of Lemma 1 allows us to restrict ourselves to the case

$$
b=\frac{1}{2}
$$

We now choose

$$
f=a_{n} \chi_{1 / 4}\left(\tau-n^{3}\right), g=b_{n} \chi_{1 / 4}\left(\tau-n^{3}\right), h=d_{n} \chi_{1 / 4}\left(\tau-n^{3}\right)
$$

where

(6.35) $a_{n}=\left\{\begin{array}{l}1, n=N, \\ 0, \text { elsewhere }\end{array} \quad b_{n}=\left\{\begin{array}{l}1, n=-N+1, \\ 0, \text { elsewhere }\end{array} \quad d_{n}=\left\{\begin{array}{l}1, n=N-1 \\ 0, \text { elsewhere }\end{array}\right.\right.\right.$

Performing the addition and integration in (6.31) in the $n_{1}, \tau_{1}$ variables respectively we find

$$
\begin{aligned}
& \sum_{n_{1}} a_{n_{1}} b_{n_{2}-n_{1}} N^{2 \rho} \int \chi_{1 / 4}\left(\tau_{1}-n_{1}^{3}\right) \chi_{1 / 4}\left(\tau_{2}-\tau_{1}-\left(n_{2}-n_{1}\right)^{3}\right) d \tau_{1} \\
& \cong c \sum_{n_{1}} a_{n_{1}} b_{n_{2}-n_{1}} N^{2 \rho} \chi_{1 / 2}\left(\tau_{2}-n_{2}^{3}+3 n_{2} n_{1}\left(n_{2}-n_{1}\right)\right) \\
& \cong c \alpha_{n_{2}} N^{2 \rho} \chi_{1 / 2}\left(\tau_{2}-1-3 N(N-1)\right),
\end{aligned}
$$

where

$$
\alpha_{n_{2}}=\left\{\begin{array}{l}
1, n_{2}=1 \\
0, \text { elsewhere }
\end{array}\right.
$$

Now performing the operations in the $n_{2}, \tau_{2}$ variables we get

$$
\begin{aligned}
& N^{3 \rho} \sum_{n_{2}} d_{n-n_{2}} \alpha_{n_{2}} \int \chi_{1 / 2}\left(\tau_{2}-1-3 N(N-1)\right) \chi_{1 / 4}\left(\tau-\tau_{2}-(N-1)^{3}\right) d \tau_{2} \\
& =c N^{3 \rho} \chi\left(\tau-N^{3}\right)
\end{aligned}
$$

By inserting this computation in (6.32) it follows that

$$
N \frac{N^{3 \rho}}{N^{\rho}} \leq c \quad \text { for any } N \in \mathbb{Z}^{+} .
$$

Hence

$$
2 \rho+1 \leq 0, \quad \text { i.e. } \quad s=-\rho \geq \frac{1}{2}
$$




\section{REFERENCES}

1. B. Birnir, C. E. Kenig, G. Ponce, N. Svanstedt and L. Vega, On the ill-posedness of the IVP for the generalized Korteweg-de Vries and nonlinear Schrödinger equations, to appear J. London Math. Soc.

2. J. L. Bona and R. Scott, Solutions of the Korteweg-de Vries equation in fractional order Sobolev spaces, Duke Math. J 43 (1976), 87-99. MR 52:14694

3. J. L. Bona and R. Smith, The initial value problem for the Korteweg-de Vries equation, Roy. Soc. London Ser A 278 (1975), 555-601. MR 52:6219

4. J. Bourgain, Fourier restriction phenomena for certain lattice subsets and applications to nonlinear evolution equations, Geometric and Functional Anal. 3 (1993), 107-156, 209-262. MR 95d:35160a,b

5. A. Cohen, Solutions of the Korteweg-de Vries equation from irregular data, Duke Math. J. 45 (1978), 149-181. MR 57:10283

6. A. Cohen and T. Kappeler, Solution to the Korteweg-de Vries equation with initial profile in $L_{1}^{1}(\mathbb{R}) \cap L_{N}^{1}\left(\mathbb{R}^{+}\right)$, SIAM J. Math. Anal. 18 (1987), 991-1025. MR 88j:35138

7. C. Fefferman, Inequalities for strongly singular convolution operators, Acta Math. 124 (1970), 9-36. MR 41:2468

8. $\_$, A note on spherical summation multipliers, Israel J. Math. 15 (1973), 44-52. MR 47:9160

9. C. S. Gardner, J. M. Greene, M. D. Kruskal and R. M. Miura, A method for solving the Korteweg-de Vries equation, Phys. Rev. Letters 19 (1967), 1095-1097.

10. Comm. Pure Appl. Math. 27 (1974), 97-133. MR 49:898

11. T. Kato, Quasilinear equations of evolutions, with applications to partial differential equation, Lecture Notes in Math. 448, Springer (1975), 27-50. MR 53:11252

12. $\quad$ On the Korteweg-de Vries equation, Manuscripta Math 29 (1979), 89-99. MR 80d:35128

13. On the Cauchy problem for the (generalized) Korteweg-de Vries equation, Advances in Mathematics Supplementary Studies, Studies in Applied Math. 8 (1983), 93-128. MR 86f: 35160

14. T. Kappeler, Solutions to the Korteweg-de Vries equation with irregular initial data, Comm. P.D.E. 11 (1986), 927-945. MR 87j:35326

15. C. E. Kenig, G. Ponce and L. Vega, Oscillatory integrals and regularity of dispersive equations, Indiana U. Math. J. 40 (1991), 33-69. MR 92d:35081

16. _ Well-posedness of the initial value problem for the Korteweg-de Vries, J. Amer. Math. Soc 4 (1991), 323-347. MR 92c:35106

17. W Well-posedness and scattering results for the generalized Korteweg-de Vries equation via the contraction principle, Comm. Pure Appl. Math. 46 (1993), 527-620. MR 94h:35229

18. _ The Cauchy problem for the Korteweg-de Vries equation in Sobolev spaces of negative indices, Duke Math. J. 71 (1993), 1-21. MR 94g:35196

19. S. Klainerman and M. Machedon, Space-time estimates for null forms and the local existence theorem, Comm. Pure Appl. Math. 46 (1993), 1221-1268. MR 94h:35137

20. __ Smoothing estimates for null forms and applications, to appear Duke Math. J.

21. D. J. Korteweg and G. de Vries, On the change of form of long waves advancing in a rectangular canal, and on a new type of long stationary waves, Philos. Mag. 539 (1895), 422-443.

22. S. N. Kruzhkov and A. V. Faminskii, Generalized solutions of the Cauchy problem for the Korteweg-de Vries equation, Math. U.S.S.R. Sbornik 48 (1984), 93-138. MR 85c:35079

23. H. Lindblad, A sharp counter example to local existence of low regularity solutions to nonlinear wave equations, Duke Math. J. 72 (1993), 503-539. MR 94h:35165

24. H. Lindblad and C. D. Sogge, On existence and scattering with minimal regularity for semilinear wave equations, preprint.

25. R. M. Miura, Korteweg-de Vries equation and generalizations. I. A remarkable explicit nonlinear transformation, J. Math. Phys. 9 (1968), 1202-1209. MR 40:6042a

26. H. Pecher, Nonlinear small data scattering for the wave and Klein-Gordon equation, Math. Z. 185 (1984), 261-270. MR 85h:35165

27. G. Ponce and T. C. Sideris, Local regularity of nonlinear wave equations in three space dimensions, Comm. P.D.E. 18 (1993), 169-177. MR 95a:35092 
28. R. L. Sachs, Classical solutions of the Korteweg-de Vries equation for non-smooth initial data via inverse scattering, Comm. P.D.E. 10 (1985), 29-89. MR 86h:35126

29. J.-C. Saut, Sur quelques généralisations de l'équations de Korteweg-de Vries, J. Math. Pures Appl. 58 (1979), 21-61. MR 82m:35133

30. J.-C. Saut and R. Temam, Remarks on the Korteweg-de Vries equation, Israel J. Math. 24 (1976), 78-87. MR 56:12676

31. A. Sjöberg, On the Korteweg-de Vries equation: existence and uniqueness, J. Math. Anal. Appl. 29 (1970), 569-579. MR 53:13885

32. R. S. Strichartz, Restriction of Fourier transforms to quadratic surface and decay of solutions of wave equations, Duke Math. J. 44 (1977), 705-714. MR 58:23577

33. S. Tanaka, Korteweg-de Vries equation: construction of solutions in terms of scattering data, Osaka J. Math. 11 (1974), 49-59. MR 50:5231

34. R. Temam, Sur un problème non linéaire, J. Math. Pures Appl. 48 (1969), 159-172. MR 41:5799

35. Y. Tsutsumi, The Cauchy problem for the Korteweg-de Vries equation with measure as initial data, SIAM J. Math. Anal. 20 (1989), 582-588. MR 90g:35153

36. A. Zygmund, On Fourier coefficients and transforms of functions of two variables, Studia Math. 50 (1974), 189-201. MR 52:8788

Department of Mathematics, University of Chicago, Chicago, Illinois 60637

E-mail address: cek@math.uchicago.edu

Department of Mathematics, University of California, Santa Barbara, California 93106

E-mail address: ponce@math.ucsb.edu

Departamento de Matematicas, Universidad del Pais Vasco, Apartado 644, 48080 BilBAO, SPAIN

E-mail address: MTPVEGOL@lg.ehu.es 